\title{
A COMPARISON PRINCIPLE AND STABILITY FOR LARGE-SCALE IMPULSIVE DELAY DIFFERENTIAL SYSTEMS
}

\author{
XINZHI LIU ${ }^{\circledR}$, XUEMIN SHEN ${ }^{2}$ and YI ZHANG ${ }^{13}$
}

(Received 6 October, 2004; revised 16 August, 2005)

\begin{abstract}
This paper studies the stability of large-scale impulsive delay differential systems and impulsive neutral systems. By developing some impulsive delay differential inequalities and a comparison principle, sufficient conditions are derived for the stability of both linear and nonlinear large-scale impulsive delay differential systems and impulsive neutral systems. Examples are given to illustrate the main results.
\end{abstract}

\section{Introduction}

Many evolution processes exhibit abrupt changes of their states at certain moments in time, such as threshold phenomena in biology, bursting rhythm models in medicine, optimal control models in economics, circuit networks and frequency modulated systems, etc. These abrupt changes are of short-term duration and may be described by impulsive differential equations. The theory of impulsive differential equations has been significantly developed in the past two decades, see $[2,5,9,11,12,15,16,20]$ and references therein. However, the corresponding theory for impulsive delay differential equations is less developed due to some theoretical and technical difficulties. Some existence and uniqueness results have been developed recently in [3] for general impulsive delay differential equations and some special classes were considered in $[1,4,8]$. Some exponential stability results for linear delay impulsive differential equations are obtained in $[1,4]$ utilising fundamental matrices. Weakly exponential stability is studied in [19]. Two criteria on asymptotic behaviour are given for a

\footnotetext{
'Department of Applied Mathematics, University of Waterloo, Waterloo, Ontario N2L 3G1, Canada; e-mail: xzliu@math.uwaterloo.ca.

${ }^{2}$ Department of Electric and Computer Engineering, University of Waterloo, Waterloo, Ontario N2L 3GI. Canada.

${ }^{3}$ China University of Petroleum, Beijing 102249, China.

(C) Australian Mathematical Society 2005, Serial-fee code 1446-1811/05
} 
nonlinear neutral differential equation with an impulse in [14]. Impulsive integrodifferential questions are studied in a Banach space in [7]. The method of Lyapunov is used to study the stability problem of impulsive delay differential equations in $[6,17,18]$. However, how to construct a suitable Lyapunov function or functional for a large-scale complex system remains a challenging issue. Recently, a new approach has been proposed in [13] for studying the stability problem of large-scale dynamic systems, where the study of a complicated large-scale system is converted to that of a lower order linear system by using a comparison principle.

In this paper, we shall study stability problems for both linear and nonlinear largescale impulsive differential equations with a time delay in the spirit of [13]. We shall first establish the comparison principle and some inequalities for impulsive delay differential equations, and then derive some sufficient conditions to guarantee stability of the nonlinear impulsive large-scale differential equations. These conditions are simple and easy to verify. Examples are given to illustrate the main results. The remainder of this paper is organised as follows. In Section 2, the comparison principle and several inequalities for linear impulsive differential equations with delay are proved, which are useful in studying the stability of large-scale impulsive delay differential equations. Some stability criteria for both linear and nonlinear large-scale impulsive delay differential equations are established in Section 3. In Section 4, the stability problem for large-scale impulsive neutral differential systems is investigated. Finally, the conclusion is given in Section 5.

\section{Preliminaries}

Let $R$ be the set of real numbers, $R^{n}$ be the space of $n$-dimensional column vectors $x=\operatorname{col}\left(x_{1}, \ldots, x_{n}\right)$ with the norm $\|x\|=\sum_{i=1}^{n}\left|x_{i}\right|$ and let $\|A\|=\max _{1 \leq j \leq m} \sum_{i=1}^{n}\left|a_{i j}\right|$ denote the norm of an $n \times m$ matrix $A=\left(a_{i j}\right)$.

Let $I=\left\{t_{k} \mid t_{1}<t_{2}<\cdots, t_{i}-t_{i-1}>\alpha>0, i=1,2, \ldots\right\}, J=\left\{t \mid t \geq t_{0}\right.$, $\left.t_{0} \in R\right\}, J_{k}=\left\{t \mid t_{k-1} \leq t<t_{k}\right\}$ and $\Delta(t)=\left\{k \mid t_{0} \leq t_{k}<t\right\}$. Without loss of generality, let $t_{0} \leq t_{1}$, where $t_{0}$ is the initial time of the IVP (initial value problem, see Sections 3-4) and $t_{1}$ is the first instant of $I$.

For $a, b \in R, a<b$, define

$$
\begin{aligned}
P C\left[[a, b], R^{n}\right]= & \left\{\phi:[a, b] \rightarrow R^{n} \mid \phi(t+0)=\phi(t), \text { for all } t \in[a, b) ;\right. \\
& \phi(t-0) \text { exists in } R^{n}, \text { for all } t \in(a, b] \text { and } \phi(t-0)=\phi(t) \\
& \text { for all but at most a finite number of points } t \in(a, b]\} ;
\end{aligned}
$$

$P C\left[[a, \infty), R^{n}\right]=\left\{\phi:[a, \infty) \rightarrow R^{n} \mid\right.$ for all $\left.b>a, \phi \in P C\left[[a, b], R^{n}\right]\right\}$.

Let $\left\|\phi_{t}\right\|=\sup _{t-\tau \leq \theta \leq t}\|\phi(\theta)\|$ denote the norm of functions $\phi \in P C\left[[\imath-\tau, t], R^{n \times m}\right]$, where $\tau>0$ is a constant. 
Let $A=\left(a_{i j}\right), B=\left(b_{i j}\right)$ be $n \times m$ matrices, denote $A \leq B$ if $a_{i j} \leq b_{i j}$ for all $i=1, \ldots, n ; j=1, \ldots, m$. Denote by $\Theta$ the zero matrix, that is, all of the entries of $\Theta$ are 0 .

LEMMA 2.1. Assume that $H \in C\left[J, R^{r \times r}\right], H=\left(h_{i j}(t)\right)_{r \times r}, h_{i j}(t) \geq 0, i \neq j$, $i, j=1,2, \ldots, r, f \in C\left[J, R^{r}\right], D_{k} \in R^{r \times r}$ and $D_{k} \geq \Theta$. Let $x, y \in C^{1}\left[J \backslash I, R^{r}\right]$ be such that

$$
\begin{cases}d x(t) / d t \leq H(t) x(t)+f(t), & t \in J \backslash I \\ x\left(t_{k}\right) \leq D_{k} x\left(t_{k}-0\right), & t_{k} \in I \\ x\left(t_{0}\right) \leq x_{0} & \end{cases}
$$

and

$$
\begin{cases}d y(t) / d t=H(t) y(t)+f(t), & t \in J \backslash I \\ y\left(t_{k}\right)=D_{k} y\left(t_{k}-0\right), & t_{k} \in I \\ y\left(t_{0}\right)=y_{0} & \end{cases}
$$

Then $x_{0} \leq y_{0}$ implies $x(t) \leq y(t)$ for $t \in J$.

PROOF. It follows from the comparison principle in [10] that $x(t) \leq y(t)$ for $t \in J_{1}$. Since $D_{1} \geq \Theta$, we get $x\left(t_{1}\right) \leq y\left(t_{1}\right)$. Let $x(t) \leq y(t), t \in\left[t_{0}, t_{k}\right)$, then $x\left(t_{k}\right) \leq y\left(t_{k}\right)$ since $D_{k} \geq \Theta$. With [10], $x(t) \leq y(t)$ for $t \in\left[t_{k}, t_{k+1}\right)$ and so $x(t) \leq y(t)$ for $t \in\left[t_{0}, t_{k+1}\right)$. By induction, $x(t) \leq y(t), t \in J$. The proof is complete.

LEMMA 2.2 (Comparison principle). Assume that $H, G \in C\left[J, R^{r \times r}\right], H(t)=$ $\left(h_{i j}(t)\right), h_{i j}(t) \geq 0, i \neq j, G(t)=\left(g_{i j}(t)\right), G \geq \Theta, f \in C\left[J, R^{r}\right]$ and that $D_{k} \geq \Theta$ are $r \times r$ matrices. Let $x, y$ be the solutions of the following systems:

$$
\begin{cases}d x(t) / d t \leq H(t) x(t)+G(t) x(t-\tau)+f(t), & t \in J \backslash I, \\ x\left(t_{k}\right) \leq D_{k} x\left(t_{k}-0\right), & t_{k} \in I, \\ x(\theta) \leq \phi(\theta), & t_{0}-\tau \leq \theta \leq t_{0}\end{cases}
$$

and

$$
\begin{cases}d y(t) / d t=H(t) y(t)+G(t) y(t-\tau)+f(t), & t \in J \backslash, \\ y\left(t_{k}\right)=D_{k} y\left(t_{k}-0\right), & t_{k} \in I, \\ y(\theta)=\psi(\theta), & t_{0}-\tau \leq \theta \leq t_{0},\end{cases}
$$

respectively, where $\phi, \psi \in P C\left[[-\tau, 0], R^{r}\right]$. Then $\phi(\theta) \leq \psi(\theta)$ implies $x(t) \leq y(t)$.

Proof. We first prove that $x(t) \leq y(t)$ for $t \in\left[t_{0}-\tau, t_{1}\right)$. 
Consider the system

$$
\begin{cases}d Y(t) / d t=H(t) Y(t)+G(t) Y(t-\tau)+f(t)+\epsilon, & t \in\left[t_{0}, t_{1}\right), \\ Y(\theta)=\psi(\theta)+\epsilon, & t_{0}-\tau \leq \theta \leq t_{0} .\end{cases}
$$

We claim that $\psi(\theta) \geq \phi(\theta)$ implies that $Y(t)>x(t)$ for $t \in\left[t_{0}-\tau, t_{1}\right)$.

In fact, if this is not true, then there exists a $t_{0}<t^{*}<t_{1}$ and some $i$ such that

$$
\begin{aligned}
x_{i}(t) & <Y_{i}(t), \quad t \in\left[t_{0}-\tau, t^{*}\right), \\
x_{i}\left(t^{*}\right) & =Y_{i}\left(t^{*}\right) \quad \text { and } \\
x_{j}(t) & \leq Y_{j}(t), \quad t \in\left[t_{0}-\tau, t^{*}\right], j \neq i .
\end{aligned}
$$

Thus $Y_{i}^{\prime}\left(t^{*}\right) \leq x_{i}^{\prime}\left(t^{*}\right)$. On the other hand,

$$
\begin{aligned}
Y_{i}^{\prime}\left(t^{*}\right) & =\sum_{j=1}^{r} h_{i j}\left(t^{*}\right) Y_{j}\left(t^{*}\right)+\sum_{j=1}^{r} g_{i j}\left(t^{*}\right) Y_{j}\left(t^{*}-\tau\right)+f_{i}\left(t^{*}\right)+\epsilon \\
& \geq \sum_{j=1}^{r} h_{i j}\left(t^{*}\right) x_{j}\left(t^{*}\right)+\sum_{j=1}^{r} g_{i j}\left(t^{*}\right) x_{j}\left(t^{*}-\tau\right)+f_{i}\left(t^{*}\right)+\epsilon \\
& >\sum_{j=1}^{r} h_{i j}\left(t^{*}\right) x_{j}\left(t^{*}\right)+\sum_{j=1}^{r} g_{i j}\left(t^{*}\right) x_{j}\left(t^{*}-\tau\right)+f_{i}\left(t^{*}\right)=x_{i}^{\prime}\left(t^{*}\right) .
\end{aligned}
$$

This contradiction indicates that $Y(t)>x(t)$ for $t_{1}>t \geq t_{0}-\tau$. Let $\epsilon \rightarrow 0$, then $y(t) \rightarrow Y(t)$ and hence $y(t) \geq x(t)$ for $t_{1}>t \geq t_{0}-\tau$.

Since $D_{1} \geq 0, x\left(t_{1}\right)=D_{1} x\left(t_{1}-0\right) \leq D_{1} y\left(t_{1}-0\right)=y\left(t_{1}\right)$. Let $x(t) \leq y(t)$ for $t \in\left[t_{0}-\tau, t_{k}\right)$, then $x\left(t_{k}\right)=D_{k} x\left(t_{k}-0\right) \leq D_{k} y\left(t_{k}-0\right)=y\left(t_{k}\right)$. Similar to the previous process, we have $x(t) \leq y(t)$ when $t \in\left[t_{0}-\tau, t_{k+1}\right)$. By induction, it follows that $x(t) \leq y(t), t \in\left[t_{0}-\tau, \infty\right)$. The proof is complete.

LEMMA 2.3. Let $A, B \in C\left[\left[t_{0}, \infty\right), R^{n \times n}\right], \Phi\left(t, t_{0}\right)$ be the fundamental matrix of $d x / d t=A(t) x$ and $x(t)=x\left(t, t_{0}, \phi\right)$ be the solution of the system

$$
\left\{\begin{array}{l}
d x / d t=A(t) x(t)+B(t) x(t-\tau) \\
x(\theta)=\phi(\theta),
\end{array} \quad \theta \in\left[t_{0}-\tau, t_{0}\right],\right.
$$

where $\phi \in P C\left[\left[t_{0}-\tau, t_{0}\right], R^{n}\right]$. Assume that there exist positive numbers $\gamma>0$ and $M>0$ such that $\left\|\Phi\left(t, t_{0}\right)\right\| \leq M e^{-\gamma\left(t-t_{0}\right)}$ and $\gamma>M \sup _{t \geq t_{0}}\|B(t)\|$. Then there exists an $\alpha>0$ such that $\|x(t)\| \leq M\left\|x_{t_{0}}\right\| e^{-\alpha\left(t-t_{0}\right)}$.

PROOF. Since $\gamma>M \sup _{t \geq t_{0}}\|B(t)\|$, there exists an $\alpha>0$ such that

$$
\gamma-\alpha-M \sup _{t \geq t_{0}}\|B(t)\| e^{\alpha \tau}>0 .
$$


We claim that, for this $\alpha,\|x(t)\| \leq M\left\|x_{t_{0}}\right\| e^{-\alpha\left(t-t_{0}\right)}$. In fact, by the method of variation of parameters, the solution of (2.1) is given by

$$
x(t)=\Phi\left(t, t_{0}\right) x\left(t_{0}\right)+\int_{t_{0}}^{t} \Phi(t, s) B(s) x(s-\tau) d s,
$$

and

$$
\begin{aligned}
\|x(t)\| & \leq\left\|\Phi\left(t, t_{0}\right)\right\|\left\|x\left(t_{0}\right)\right\|+\int_{t_{0}}^{t}\|\Phi(t, s)\|\|B(s)\|\|x(s-\tau)\| d s \\
& \leq M e^{-\gamma\left(t-t_{0}\right)}\left\|x_{t_{0}}\right\|+\int_{t_{0}}^{t} M e^{-\gamma(t-s)}\|B(s)\|\|x(s-\tau)\| d s .
\end{aligned}
$$

Let $Q(t)=e^{\alpha\left(t-t_{0}\right)}\left[M e^{-\gamma\left(t-t_{0}\right)}\left\|x_{t_{0}}\right\|+\int_{t_{0}}^{t} M e^{-r(t-s)}\|B(s)\|\|x(s-\tau)\| d s\right], t \geq t_{0}$ and $Q(t)=\left\|x_{t_{0}}\right\|, t \in\left[t_{0}-\tau, t_{0}\right]$. Then

$$
Q(t) \geq\|x(t)\| e^{\alpha\left(t-t_{0}\right)}, \quad t \geq t_{0}-\tau
$$

and

$$
\begin{aligned}
Q^{\prime}(t)= & \alpha e^{\alpha\left(t-t_{0}\right)}\left[M e^{-\gamma\left(t-t_{0}\right)}\left\|x_{t_{0}}\right\|+s \int_{t_{0}}^{t} M e^{-\gamma(t-s)}\|B(s)\|\|x(s-\tau)\| d s\right] \\
& +e^{\alpha\left(t-t_{0}\right)}\left[-\gamma M e^{-\gamma\left(t-t_{0}\right)}\left\|x_{t_{0}}\right\|-\gamma \int_{t_{0}}^{t} M e^{-\gamma(t-s)}\|B(s)\|\|x(s-\tau)\| d s\right] \\
& +M e^{\alpha\left(t-t_{0}\right)}\|B(t)\|\|x(t-\tau)\| \\
= & \alpha Q(t)-\gamma Q(t)+M e^{\alpha\left(t-t_{0}\right)}\|B(t)\|\|x(t-\tau)\| \\
= & (\alpha-\gamma) Q(t)+M e^{\alpha\left(t-t_{0}\right)}\|B(t)\|\|x(t-\tau)\| \\
\leq & (\alpha-\gamma) Q(t)+M\|B(t)\|\|Q(t-\tau)\| e^{\alpha \tau}
\end{aligned}
$$

in view of (2.2).

For any $K>1$, we claim that $Q(t)<K\left\|Q_{t_{0}}\right\|=: L, L>0, t \geq t_{0}-\tau$. If this is not true, then there exists $t^{*}>t_{0}$ such that $Q\left(t^{*}\right)=L, Q(t)<L, t_{0}-\tau \leq t<t^{*}$ and $Q^{\prime}\left(t^{*}\right) \geq 0$. On the other hand,

$$
\begin{aligned}
Q^{\prime}\left(t^{*}\right) & \leq(\alpha-\gamma) Q\left(t^{*}\right)+M\left\|B\left(t^{*}\right)\right\|\left\|Q\left(t^{*}-\tau\right)\right\| e^{\alpha \tau} \\
& \leq(\alpha-\gamma) L+M\left\|B\left(t^{*}\right)\right\| L e^{\alpha \tau} \\
& \leq\left(\alpha-\gamma+M\left\|B\left(t^{*}\right)\right\| e^{\alpha \tau}\right) L<0 .
\end{aligned}
$$

This contradiction implies $Q(t)<L$. Let $K \rightarrow 1$, then $Q(t) \leq\left\|Q_{t_{0}}\right\|$ and

$$
\|x(t)\| \leq Q(t) e^{-\alpha\left(t-t_{0}\right)} \leq\left\|Q_{t_{0}}\right\| e^{-\alpha\left(t-t_{0}\right)}=M\left\|x_{t_{0}}\right\| e^{-\alpha\left(t-t_{0}\right)}, \quad t \geq t_{0}-\tau .
$$

The proof is complete. 
LEMMA 2.4. Let $A, B \in C\left[\left[t_{0}, \infty\right), R^{r \times r}\right]$ and $\Phi\left(t, t_{0}\right)$ be the fundamental matrix of $d x / d t=A(t) x(t)$. Assume that

(1) there exist positive numbers $\gamma>0$ and $M>0$ such that $\left\|\Phi\left(t, t_{0}\right)\right\| \leq M e^{-\gamma\left(t-t_{0}\right)}$ and $\gamma>M \sup _{t \geq 1_{0}}\|B(t)\|$;

(2) $x\left(t, t_{0}, \phi\right)$ is the solution of the IVP

$$
\begin{cases}d x(t) / d t=A(t) x(t)+B(t) x(t-\tau), & t \in J \backslash I, \\ x\left(t_{k}\right)=D_{k} x\left(t_{k}-0\right), & t_{k} \in I, \\ x(\theta)=\phi(\theta), & t_{0}-\tau \leq \theta \leq t_{0},\end{cases}
$$

where $\phi \in P C\left[\left[t_{0}-\tau, t_{0}\right], R^{n}\right]$ and $t_{k+1}-t_{k} \geq \tau$.

Then there exists an $\alpha>0$ such that

$$
\|x(t)\| \leq\left\|x_{t_{0}}\right\| \prod_{j \in \Delta(t)} M^{k+1} \max \left\{\left\|D_{j}\right\|, e^{\alpha \tau}\right\} e^{-\alpha\left(t-t_{0}\right)}, \quad t_{k} \leq t<t_{k+1} .
$$

PROOF. From Lemma 2.3, it follows that for any $k$, we have

$$
\|x(t)\| \leq M\left\|x_{t_{k}}\right\| e^{-\alpha\left(t-t_{k}\right)}, \quad t \in\left[t_{k}, t_{k+1}\right) .
$$

Since $x\left(t_{k}\right)=D_{k} x\left(t_{k}-0\right)$, it follows that

$$
\begin{aligned}
\left\|x_{t_{k}}\right\| & =\sup _{t_{k}-\tau \leq 1 \leq t_{k}}\|x(t)\| \\
& \leq \max \left\{\sup _{t_{k}-\tau \leq t<t_{k}}\|x(t)\|,\left\|x\left(t_{k}\right)\right\|\right\} \\
& \leq \max \left\{\sup _{t_{k}-\tau \leq t<t_{k}}\|x(t)\|,\left\|D_{k}\right\|\left\|x\left(t_{k}-0\right)\right\|\right\} \\
& \leq \max \left\{\sup _{t_{k}-t \leq t<t_{k}} M\left\|x_{t_{k-1}}\right\| e^{-\alpha\left(t-t_{k-1}\right)}, M\left\|D_{k}\right\|\left\|x_{t_{k-1}}\right\| e^{-\alpha\left(t_{k}-t_{k-1}\right)}\right\} \\
& \leq M\left\|x_{t_{t-1}}\right\| e^{-\alpha\left(t_{k}-t_{k-1}\right)} \max \left\{e^{\alpha \tau},\left\|D_{k}\right\|\right\} .
\end{aligned}
$$

Using a similar argument, we have $\left\|x_{t_{t-1}}\right\| \leq M\left\|x_{t_{k-2}}\right\| e^{-\alpha\left(t_{k-1}-t_{k-2}\right)} \max \left\{e^{\alpha \tau},\left\|D_{k-1}\right\|\right\}$ and so on. Thus we get

$$
\begin{aligned}
\|x(t)\| & \leq M\left\|x_{t_{k}}\right\| e^{-\alpha\left(t-t_{k}\right)} \\
& \leq M^{2}\left\|x_{t_{k-1}}\right\| e^{-\alpha\left(t-t_{k-1}\right)} \max \left\{e^{\alpha \tau},\left\|D_{k}\right\|\right\} \\
& \leq \cdots \leq\left\|x_{t_{0}}\right\| \prod_{j \in \Delta(t)} M^{k+1} \max \left\{\left\|D_{j}\right\|, e^{\alpha \tau}\right\} e^{-\alpha\left(t-t_{0}\right)}, \quad t_{k} \leq t<t_{k+1} .
\end{aligned}
$$

The proof is complete. 


\section{Stability of large-scale impulsive delay systems}

Consider the large-scale impulsive delay differential equations

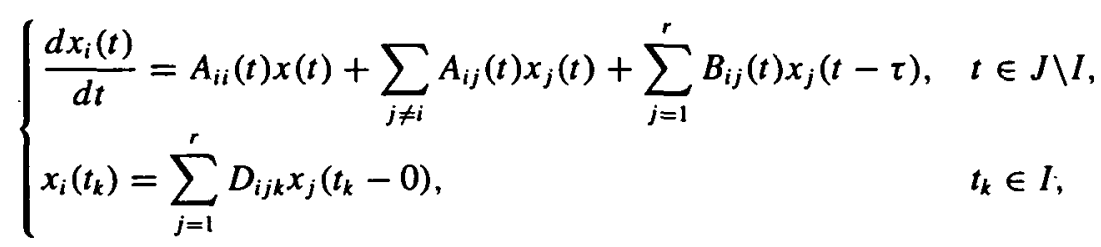

where $x_{i}=\left(x_{1}^{i}, \ldots, x_{n_{i}}^{i}\right)^{T} \in R^{n_{i}}, A_{i j}, B_{i j} \in C\left[J, R^{n_{i} \times n_{j}}\right], D_{i j k} \in R^{n_{i} \times n_{j}}, i, j=$ $1, \ldots, r, k=1,2, \ldots$ and $\sum_{i=1}^{r} n_{i}=n$.

In this section and in the following section, we always assume that $t_{k}-t_{k-1} \geq \tau$, $k=1,2, \ldots$.

Assume that there exist positive numbers $\alpha_{i}>0, c_{i}>0, i=1, \ldots, r$ such that the fundamental solution matrix $R_{i}\left(t, t_{0}\right)$ of the isolated subsystem

$$
d x_{i}(t) / d t=A_{i i}(t) x_{i}(t)
$$

satisfies $\left\|R_{i}\left(t, t_{0}\right)\right\| \leq c_{i} e^{-\alpha_{i}\left(t-t_{0}\right)}$ and

$$
\begin{array}{rlrl}
\left\|A_{i j}(t)\right\| & \leq a_{i j}(t)<+\infty, & & i, j=1,2, \ldots, r, i \neq j, \\
\left\|B_{i j}(t)\right\| & \leq b_{i j}(t)<+\infty, & i, j=1,2, \ldots, r \text { and } \\
\left\|D_{i j k}\right\| & \leq d_{i j k}, & i, j=1,2, \ldots, r, k=1,2, \ldots
\end{array}
$$

Denote by $\tilde{A}(t)=\left(\left(1-\delta_{i j}\right) c_{i} a_{i j}(t)\right)_{r \times r}, B(t)=\left(c_{i} b_{i j}(t)\right)_{r \times r}$ and $\tilde{D}_{k}=\left(d_{i j k}\right)_{r \times r}$, where

$$
\delta_{i j}= \begin{cases}1, & i=j \\ 0, & i \neq j\end{cases}
$$

We are ready to state and prove our first result.

THEOREM 3.1. Assume that $-\alpha_{j}+\sum_{i \neq j} c_{i} a_{i j}(t)<-\gamma<0, j=1, \ldots, r,-\gamma+$ $\sup _{t \geq t_{0}}\|\tilde{B}(t)\|<0$ and $\alpha>0$ is the solution of $\gamma-\sup _{t \geq t_{0}}\|\tilde{B}(t)\| e^{\alpha \tau}-\alpha>0$. Then

(1) $\lim \sup _{t \rightarrow \infty}\left(\prod_{j \in \Delta(t)} \max \left\{\left\|\tilde{D}_{j}\right\|, e^{\alpha \tau}\right\}\right) / e^{\alpha\left(t-t_{0}\right)}<\infty$ implies system (3.1) is stable;

(2) $\lim \sup _{t \rightarrow \infty}\left(\prod_{j \in \Delta(t)} \max \left(\left\|\tilde{D}_{j}\right\|, e^{\alpha \tau}\right\}\right) / e^{\alpha\left(t-t_{0}\right)}=0$ implies system (3.1) is asymptotically stable; 
(3) if there exists a positive number $\beta$, such that $\beta<\alpha$ and

$$
\limsup _{t \rightarrow \infty} \frac{\prod_{j \in \Delta(t)} \max \left\{\left\|\tilde{D}_{j}\right\|, e^{\alpha \tau}\right\}}{e^{\beta\left(t-t_{0}\right)}}<\infty,
$$

then system (3.1) is exponentially stable.

PROOF. From (3.1), we have, for $t \in J_{k+1}$,

$$
\begin{aligned}
x_{i}(t)= & R_{i}\left(t, t_{k}\right) x_{i}\left(t_{k}\right) \\
& +\int_{t_{k}}^{t}\left[R_{i}(t, s) \sum_{j \neq i} A_{i j}(s) x_{j}(s)+R_{i}(t, s) \sum_{j=1}^{r} B_{i j}(s) x_{j}(s-\tau)\right] d s
\end{aligned}
$$

and

$$
\begin{aligned}
\left\|x_{i}(t)\right\| \leq & c_{i} e^{-\alpha_{i}\left(t-t_{k}\right)}\left\|x_{i}\left(t_{k}\right)\right\|+\int_{t_{k}}^{t} c_{i} e^{-\alpha_{i}(t-s)} \sum_{j \neq i} a_{i j}(s)\left\|x_{j}(s)\right\| d s \\
& +\int_{t_{k}}^{t} c_{i} e^{-\alpha_{i}(t-s)} \sum_{j=1}^{r} b_{i j}(s)\left\|x_{j}(s-\tau)\right\| d s=: P_{i}(t) .
\end{aligned}
$$

Then $\left\|x_{i}(t)\right\| \leq P_{i}(t), t \in J_{k+1}$, and

$$
P_{i}^{\prime}(t) \leq-\alpha_{i} P_{i}(t)+\sum_{j \neq i} c_{i} a_{i j}(t) P_{j}(t)+\sum_{j=1}^{r} c_{i} b_{i j}(t) P_{j}(t-\tau), \quad t \in J \backslash I .
$$

Let $P=\operatorname{col}\left(P_{1}, \ldots, P_{r}\right), P\left(t_{k}\right)=\tilde{D}_{k} P\left(t_{k}-0\right), t_{k} \in I$, then $\| x_{i}(t) \mid \leq P_{i}(t), t \in J$.

Consider the comparison system

$$
\begin{cases}P^{\prime}(t) \leq \operatorname{diag}\left(-\alpha_{1}, \ldots,-\alpha_{r}\right) P(t)+\tilde{A}(t) P(t)+\tilde{B}(t) P(t-\tau), & t \in J \backslash I, \\ P\left(t_{k}\right)=\tilde{D}_{k} P\left(t_{k}-0\right), & t_{k} \in I ;\end{cases}
$$

and

$$
\begin{cases}\xi^{\prime}(t)=\operatorname{diag}\left(-\alpha_{1}, \ldots,-\alpha_{r}\right) \xi(t)+\tilde{A}(t) \xi(t)+\tilde{B}(t) \xi(t-\tau), & t \in J \backslash I, \\ \xi\left(t_{k}\right)=\tilde{D}_{k} \xi\left(t_{k}-0\right), & t_{k} \in I,\end{cases}
$$

where $\xi(t)=\operatorname{col}\left(\xi_{1}(t), \ldots, \xi_{r}(t)\right)$. Since $-\alpha_{j}+\sum_{i \neq j} c_{i} a_{i j}(t)<-\gamma<0$, we claim that the solution $\eta(t)$ of the system

$$
\eta^{\prime}(t)=\operatorname{diag}\left(-\alpha_{1}, \ldots,-\alpha_{r}\right) \eta(t)+\tilde{A}(t) \eta(t)
$$

satisfies $|\eta(t)| \leq\left\|\eta\left(t_{0}\right)\right\| e^{-r\left(t-t_{0}\right)}$ and hence the fundamental matrix $\Phi\left(t, t_{0}\right)$ of (3.3) satisfies $\left\|\Phi\left(t, t_{0}\right)\right\| \leq e^{-\gamma\left(t-t_{0}\right)}$, where $\eta=\operatorname{col}\left(\eta_{1}, \ldots, \eta_{r}\right)$. 
In fact, let $Q(t)=\sum_{i=1}^{n}\left|\eta_{i}(t)\right|, t \geq t_{0}$, then $Q\left(t_{0}\right)=\sum_{i=1}^{n}\left|\eta_{i}\left(t_{0}\right)\right|$ and

$$
\begin{aligned}
D^{+} Q(t) & =\sum_{i=1}^{n} D^{+}\left|\eta_{i}(t)\right| \leq \sum_{i=1}^{n}\left[-\alpha_{i}\left|\eta_{i}(t)\right|+\sum_{j \neq i}^{n} c_{i} a_{i j}(t)\left|\eta_{j}(t)\right|\right] \\
& =\sum_{j=1}^{n}\left(-\alpha_{j}\left|\eta_{j}(t)\right|\right)+\sum_{j=1}^{n}\left(\sum_{i \neq j}^{n} c_{i} a_{i j}(t)\right)\left|\eta_{j}(t)\right| \\
& =\sum_{j=1}^{n}\left(-\alpha_{j}+\sum_{i \neq j}^{n} c_{i} a_{i j}(t)\right)\left|\eta_{j}(t)\right| \leq-\gamma \sum_{j=1}^{n}\left|\eta_{j}(t)\right|=-\gamma Q(t) .
\end{aligned}
$$

Thus

$$
\|\eta(t)\|=\sum_{i=1}^{n}\left|\eta_{i}(t)\right|=Q(t) \leq Q\left(t_{0}\right) e^{-\gamma\left(t-t_{0}\right)}=\left\|\eta\left(t_{0}\right)\right\| e^{-\gamma\left(t-t_{0}\right)}
$$

and so $\left\|\Phi\left(t, t_{0}\right)\right\| \leq e^{-\gamma\left(t-t_{0}\right)}, t \geq t_{0}$, since $\Phi\left(t, t_{0}\right)$ is the fundamental matrix.

From Lemma 2.4 and the condition $-\gamma+\sup _{t \geq t_{0}}\|\tilde{B}(t)\|<0$, it follows that there exists an $\alpha>0$ such that the solutions of system (3.2) satisfy

$$
\left\|\xi\left(t, t_{0}, \xi_{t_{0}}\right)\right\| \leq\left\|\xi_{t_{0}}\right\| \prod_{j \in \Delta(t)} \max \left\{\left\|\tilde{D}_{j}\right\|, e^{\alpha \tau}\right\} e^{-\alpha\left(t-t_{0}\right)}
$$

It follows from Lemma 2.2 that $\left\|x_{i}(t)\right\| \leq P_{i}(t) \leq \xi_{i}(t), i=1, \ldots, r$, and from (3.4) that statements (1)-(3) of Theorem 3.1 are true. The proof is complete.

COROLLARY 3.2. Assume that the conditions of Theorem 3.1 hold and there exist positive numbers $\eta>\tau$ and $M>0$ such that $t_{k}-t_{k-1}=\eta$ for all $k=1,2, \ldots$ and $\max \left\{\sup \left\{\left\|\tilde{D}_{k}\right\|\right\}, e^{\alpha \tau}\right\} \leq M$. Then $M<e^{\alpha \eta}$ implies system (3.1) is exponentially stable.

Proof. In this case

$$
\prod_{j \in \Delta(t)} \max \left\{\left\|\tilde{D}_{j}\right\|, e^{\alpha \tau}\right\}=e^{\left.\sum_{j \in \Delta(t)} \ln \max \mid\left\|\tilde{D}_{j}\right\|, e^{\alpha \tau}\right)} \leq e^{k \ln M}, \quad t \in\left[t_{k}, t_{k+1}\right)
$$

and so

$$
\frac{\prod_{j \in \Delta(t)} \max \left\{\left\|\tilde{D}_{j}\right\|, e^{\alpha \tau}\right\}}{e^{\alpha\left(t-t_{0}\right)}} \leq e^{k \ln M-\alpha\left(t-t_{0}\right)} \leq e^{k \ln M-k \alpha \eta},
$$

which implies the required result. 
COROLlaRY 3.3. Assume that the conditions of Theorem 3.1 hold and there exist positive numbers $M>0$ and $p>0$ such that $\max \left\{\sup \left\{\left\|\tilde{D}_{k}\right\|\right\}, e^{\alpha \tau}\right\} \leq M$ and

$$
\lim _{T \rightarrow \infty} \frac{\eta(t, t+T)}{T}=p,
$$

where $\eta\left(t_{0}, t\right)$ denotes the number of impulses in the time interval $\left[t_{0}, t\right)$.

Then $M<e^{\alpha / p}$. implies that system (3.1) is exponentially stable.

Proof. From the condition, it follows that for any $\epsilon>0$, there exists $T>0$ and $\tilde{M}=\tilde{M}\left(t_{0}\right)>0$, such that when $t \geq t_{0}+T$,

$$
\prod_{j \in \Delta(t)} \max \left\{\left\|\tilde{D}_{j}\right\|, e^{\alpha \tau}\right\}=e^{\left.\sum_{j \in \Delta(t)} \ln \max \left\|\tilde{D}_{j}\right\|, e^{\alpha \tau}\right\}} \leq e^{\frac{n(t 0, t)}{1-t_{0}}\left(t-t_{0}\right) \ln M} \leq \tilde{M} e^{(p+\epsilon) \ln M\left(t-t_{0}\right)}
$$

and so

$$
\frac{\prod_{j \in \Delta(t)} \max \left\{\left\|\tilde{D}_{j}\right\|, e^{\alpha \tau}\right\}}{e^{\alpha\left(t-t_{0}\right)}} \leq \tilde{M} e^{[(p+\epsilon) \ln M-\alpha]\left(t-t_{0}\right)} .
$$

Since $\epsilon$ can be chosen arbitrarily small, (3.5) implies the required results.

EXAMPLE 1. Consider the large-scale impulsive delay system

$$
\left\{\begin{aligned}
d x_{i}(t) / d t=A_{i 1}(t) x_{1}(t)+A_{i 2}(t) x_{2}(t) & \\
+B_{i 1}(t) x_{1}(t-\tau)+B_{i 2}(t) x_{2}(t-\tau), & i=1,2, t \in J \backslash I, \\
x_{i}\left(t_{k}\right)=D_{i 1}\left(t_{k}\right) x_{1}\left(t_{k}-0\right)+D_{i 2}\left(t_{k}\right) x_{2}\left(t_{k}-0\right), & i=1,2, t_{k} \in I,
\end{aligned}\right.
$$

where $x_{1}, x_{2} \in R^{2}, A_{i j}, B_{i j}, D_{i j} \in R^{2 \times 2}, i, j=1,2$.

Let $t_{0}=0, \tau=1$,

$$
A_{11}(t)=\left[\begin{array}{cc}
-4 & 0 \\
\frac{1}{2} \sin ^{2} t & -4
\end{array}\right] \text { and } A_{22}(t)=\left[\begin{array}{cc}
-4+\frac{3}{4} \sin t & 0 \\
0 & -3
\end{array}\right]
$$

Choose $A_{12}(t), A_{21}(t)$ and $B(t)=\left(B_{i j}(t)\right)$ such that $\left\|A_{12}(t)\right\|<1-\left(4 e^{2}\right)^{-1}$, $\left\|A_{21}(t)\right\|<1-\left(4 e^{2}\right)^{-1}$ and $\|B(t)\| \leq e^{-1}$. Then $\alpha_{i}=-3, c_{i}=1, i=1,2$. Choose $\gamma>2, \alpha=1$ and so $\gamma-\sup _{t \geq 1_{0}}\|\tilde{B}(t)\| e^{\alpha \tau}-\alpha>0$. Let

$$
D_{k}=\left[\begin{array}{cc}
D_{11 k} & D_{12 k} \\
D_{21 k} & D_{22 k}
\end{array}\right]=\left[\begin{array}{cccc}
e-3 / 2 & -1 / 2 & 0 & 1 \\
1 & 0 & 0 & 0 \\
0 & 1 & 0 & -1 \\
0 & 1 / 2 & 0 & 0
\end{array}\right] \text {, }
$$

then $\left\|D_{k}\right\|=\left\|\tilde{D}_{k}\right\|=e-1 / 2$ and if 
(1) $t_{k}-t_{k-1} \geq \tau=1$, then $\left(\prod_{j \in \Delta(t)} \max \left\{\left\|\tilde{D}_{j}\right\|, e^{\alpha \tau}\right\}\right) / e^{t} \leq 1$,

(2) $t_{k}-t_{k-1} \geq \tau+1 / k$, then $\lim _{t \rightarrow \infty}\left(\left(\prod_{j \in \Delta(t)} \max \left\{\left\|\tilde{D}_{j}\right\|, e^{\alpha \tau}\right\}\right) / e^{\alpha t}=0\right.$,

(3) $t_{k}-t_{k-1} \geq \tau+\eta, \eta>0$, then $\left(\prod_{j \in \Delta(l)} \max \left\{\left\|\tilde{D}_{j}\right\|, e^{\alpha \tau}\right\}\right) / e^{t /(1+\eta)} \leq 1$.

Thus we can conclude that system (3.6) is: stable if $t_{k}-t_{k-1} \geq \tau=1$; asymptotically stable if $t_{k}-t_{k-1} \geq \tau+1 / k$; and exponentially stable if $t_{k}-t_{k-1} \geq \tau+\eta, \eta>0$.

REMARK. This example illustrates that Theorem 3.1 is simple and easily verified. It is very interesting to notice that, from the example, even if at every impulsive point $t_{k}$, $\left\|D_{k}\right\|>1$, which implies that the norm of solutions is increased at impulsive points, that the system may still be stable or exponentially stable.

Consider the nonlinear impulsive delay differential system

$$
\begin{cases}d x / d t=\operatorname{diag}\left(A_{11}(t), \ldots, A_{r r}(t)\right) x(t)+F(t, x(t), x(t-\tau)), & t \in J \backslash I, \\ x\left(t_{k}\right)=D_{k} x\left(t_{k}-0\right), & t_{k} \in I,\end{cases}
$$

where $F \in C\left[I \times R^{n} \times R^{n}, R^{n}\right], F(t, 0,0) \equiv 0, x=\left(x_{1}, \ldots, x_{r}\right)^{T} \in R^{n}, x_{i} \in R^{n_{i}}$, $A_{i i}(t)$ are $n_{i} \times n_{i}$ matrices, $D_{k}=\left(D_{i j k}\right)$ are $n \times n$ matrices, $D_{i j k}$ are $n_{i} \times n_{j}$ matrices, $i, j=1,2, \ldots, r, \sum_{i=1}^{r} n_{i}=n, k=1,2, \ldots$ Rewriting (3.7) by components,

$$
\begin{cases}d x_{i} / d t=A_{i i}(t) x_{i}(t)+F_{i}(t, x(t), x(t-\tau)), & t \in J \backslash I, \\ x_{i}\left(t_{k}\right)=\sum_{j=1}^{r} \cdot D_{i j k} x_{j}\left(t_{k}-0\right), & t_{k} \in I, i=1,2, \ldots, r .\end{cases}
$$

THEOREM 3.4. If

(1) there exist scalar functions $l_{i j}, k_{i j} \in C[J, R]$, such that

$$
\left\|F_{i}(t, x, y)\right\| \leq \sum_{j=1}^{r} l_{i j}(t)\left\|x_{j}\right\|+\sum_{j=1}^{r} k_{i j}(t)\left\|y_{j}\right\|, \quad i=1,2, \ldots, r
$$

(2) there exist constants $c_{i}$ and scalar functions $\beta_{i} \in C[J, R]$, such that the fundamental solution matrix $R_{i i}\left(t, t_{0}\right)$ of the isolated subsystem $d x_{i} / d t=A_{i i}(t) x_{i}(t)$ satisfies $\left\|R_{i i}\left(t, t_{0}\right)\right\| \leq c_{i} e^{-\int_{t_{0}}^{\prime} \beta_{i}(s) d s}, i=1, \ldots, r$,

then the stability, uniform stability, global asymptotic stability, global uniform asymptotic stability, global exponential stability, the Lagrange stability of all solutions, uniform Lagrange stability of the trivial solution of the lower dimension linear equations

$$
\begin{cases}\frac{d \eta_{i}(t)}{d t}=-\beta_{i}(t) \eta_{i}(t)+\sum_{j=1}^{r} c_{i} l_{i j}(t) \eta_{j}(t)+\sum_{j=1}^{r} c_{i} k_{i j}(t) \eta_{j}(t-\tau), & t \in J \backslash I, \\ \eta_{i}\left(t_{k}\right)=\sum_{j=1}^{r}\left\|D_{i j k}\right\| \eta_{j}\left(t_{k}-0\right), & t_{k} \in I,\end{cases}
$$


where $i=1, \ldots, r$, imply the stability, uniform stability, global asymptotic stability, global uniform asymptotic stability, global exponential stability, the Lagrange stability of all solutions, uniform Lagrange stability of the high dimension nonlinear system (3.7), respectively.

PROOF. Let $x(t)=x\left(t, t_{0}, \phi\right)$ be the solution of (3.7) satisfying the initial condition $x(t)=\phi(t), t_{0}-\tau \leq t \leq t_{0}$. Then we have, for $i=1, \ldots, r$ and $t \in J_{k+1}$,

$$
\left\{\begin{array}{l}
x_{i}(t)=R_{i}\left(t, t_{k}\right) x_{i}\left(t_{k}\right)+\int_{t_{k}}^{t} R_{i}(t, s) F_{i}(s, x(s), x(s-\tau)) d s, \\
x_{i}\left(t_{k}\right)=\sum_{j=1}^{r} D_{i j k} x_{j}\left(t_{k}-0\right)
\end{array}\right.
$$

and

$$
\left\{\begin{aligned}
\left\|x_{i}(t)\right\| \leq & c_{i} e^{-\int_{t_{k}}^{\prime} \beta_{i}(s) d s}\left\|x_{i}\left(t_{k}\right)\right\| \\
& +\sum_{j=1}^{r} \int_{t_{k}}^{t} c_{i} e^{-\int_{s}^{t} \beta_{i}(v) d v}\left[l_{i j}(s)\left\|x_{j}(s)\right\|+k_{i j}(s)\left\|x_{j}(s-\tau)\right\|\right] d s, \\
\left\|x_{i}\left(t_{k}\right)\right\| \leq & \sum_{j=1}^{r}\left\|D_{i j k}\right\|\left\|x_{j}\left(t_{k}-0\right)\right\| .
\end{aligned}\right.
$$

Let for $i=1, \ldots, r$ and $t \in J_{k+1}$,

$$
\left\{\begin{aligned}
\xi_{i}(t)= & c_{i} e^{-\int_{i_{k}}^{\prime} \beta_{j}(s) d s}\left\|x_{i}\left(t_{k}\right)\right\| \\
& +\sum_{j=1}^{r} \int_{t_{k}}^{t} c_{i} e^{-\int_{s}^{t} \beta_{i}(v) d v}\left[l_{i j}(s)\left\|x_{j}(s)\right\|+k_{i j}(s)\left\|x_{j}(s-\tau)\right\|\right] d s, \\
\xi_{i}\left(t_{k}\right)= & \sum_{j=1}^{r}\left\|D_{i j k}\right\| \xi_{j}\left(t_{k}-0\right),
\end{aligned}\right.
$$

then $\left\|x_{i}(t)\right\| \leq \xi_{i}(t), i=1, \ldots, r$, and

$$
\left\{\begin{array}{l}
\frac{d \xi_{i}(t)}{d t} \leq-\beta_{i}(t) \xi_{i}(t)+\sum_{j=1}^{r} c_{i} l_{i j}(t) \xi_{j}(t)+\sum_{j=1}^{r} c_{i} k_{i j}(t) \xi_{j}(t-\tau), \\
\xi_{i}\left(t_{k}\right)=\sum_{j=1}^{r}\left\|D_{i j k}\right\| \xi_{j}\left(t_{k}-0\right) .
\end{array}\right.
$$

With the comparison system for $i=1, \ldots, r$ and $t \in J_{k+1}$,

$$
\left\{\begin{array}{l}
\frac{d \eta_{i}}{d t}=-\beta_{i}(t) \eta_{i}(t)+\sum_{j=1}^{r} c_{i} l_{i j}(t) \eta_{j}(t)+\sum_{j=1}^{r} c_{i} k_{i j}(t) \eta_{j}(t-\tau), \\
\eta_{i}\left(t_{k}\right)=\sum_{j=1}^{r}\left\|D_{i j k}\right\| \eta_{j}\left(t_{k}-0\right),
\end{array}\right.
$$

and from Lemma 2.2, we have $\left\|x_{i}(t)\right\| \leq \xi_{i}(t) \leq \eta_{i}(t), t \geq t_{0}, i=1, \ldots, r$. The inequality implies the results of the theorem. The proof is complete. 
EXAMPLE 2. Consider the nonlinear impulsive delay differential system

$$
\left\{\begin{aligned}
\frac{d x_{1}}{d t}= & {\left[\begin{array}{cc}
-5+e^{-t} & \ln \left(1+t^{2}\right) \\
-\ln \left(1+t^{2}\right) & -4
\end{array}\right] x_{1}+\frac{|\sin t|}{2 \sqrt{2}\left(1+\left\|x_{2}\right\|\right)} x_{2} } \\
& +\frac{\left(1+\sin t^{2}\right) \sin ^{2}\left(x_{1}^{T} x_{2}\right)}{4 \sqrt{2} e^{2}} x_{1}(t-1), \\
\frac{d x_{2}}{d t}= & {\left[\begin{array}{cc}
-6+\sin t & t \\
-t & -7+2 \cos ^{2} t
\end{array}\right] x_{2}+\frac{\sqrt{2}}{3\left(1+\ln \left(1+\left\|x_{2}\right\|^{2}\right)\right)} x_{1} } \\
& +\frac{\sqrt{2}}{4 e^{2}\left(2-e^{\left.-t\left\|x_{1}(t-1)\right\|^{2}\right)}\right.} x_{2}(t-1), \quad t \neq t_{k}, \\
x\left(t_{k}\right)= & D_{k} x\left(t_{k}-0\right), \quad k=1,2, \ldots,
\end{aligned}\right.
$$

where $x=\left(x_{1}^{T}, x_{2}^{T}\right)^{T} \in R^{4}, x_{1}, x_{2} \in R^{2}, D_{k}=\left(D_{i j k}\right) \in R^{4 \times 4}$ and $D_{i j k} \in R^{2 \times 2}$, $i, j=1,2$.

Using the notation of Theorem 3.4, we have

$$
\begin{gathered}
A_{11}=\left[\begin{array}{cc}
-5+e^{-t} & \ln \left(1+t^{2}\right) \\
-\ln \left(1+t^{2}\right) & -4
\end{array}\right], \quad A_{22}=\left[\begin{array}{cc}
-6+\sin t & t \\
-t & -7+2 \cos ^{2} t
\end{array}\right], \\
F_{1}(t, x, x(t-\tau))=\frac{|\sin t|}{2 \sqrt{2}\left(1+\left\|x_{2}\right\|\right)} x_{2}+\frac{\left(1+\sin t^{2}\right) \sin ^{2}\left(x_{1}^{T} x_{2}\right)}{4 \sqrt{2} e^{2}} x_{1}(t-1) \quad \text { and } \\
F_{2}(t, x, x(t-\tau))=\frac{\sqrt{2}}{3\left(1+\ln \left(1+\left\|x_{2}\right\|^{2}\right)\right)} x_{1}+\frac{\sqrt{2}}{4 e^{2}\left(2-e^{\left.-t\left\|x_{1}(t-1)\right\|^{2}\right)}\right.} x_{2}(t-1) .
\end{gathered}
$$

By choosing $c_{i}=\sqrt{2}, \beta_{i}=4, l_{11}(t)=l_{22}(t)=k_{12}(t)=k_{21}(t)=0, l_{12}(t)=\sqrt{2} / 4$, $l_{21}(t)=\sqrt{2} / 3, k_{11}=\left(2 \sqrt{2} e^{2}\right)^{-1}$ and $k_{22}(t)=\left(2 \sqrt{2} e^{2}\right)^{-1}$, the comparison system is

$$
\left\{\begin{array}{rlrl}
\frac{d \eta_{1}}{d t} & =-4 \eta_{1}(t)+\frac{1}{2} \eta_{2}(t)+\frac{1}{2 e^{2}} \eta_{1}(t-1), & & \\
\frac{d \eta_{2}}{d t} & =-4 \eta_{2}(t)+\frac{2}{3} \eta_{1}(t)+\frac{1}{2 e^{2}} \eta_{2}(t-1), & & t \neq t_{k}, k=1, \ldots, \\
\eta_{i}\left(t_{k}\right) & =\sum_{j=1}^{2}\left\|D_{i j k}\right\| \eta_{j}\left(t_{k}-0\right), & i=1,2, k=1, \ldots
\end{array}\right.
$$

For system (3.11), using the notation of Theorem 3.1,

$$
A=\left[\begin{array}{ll}
A_{11} & A_{12} \\
A_{21} & A_{22}
\end{array}\right]=\left[\begin{array}{cc}
-4 & 1 / 2 \\
2 / 3 & -4
\end{array}\right], \quad B=\left[\begin{array}{ll}
B_{11} & B_{12} \\
B_{21} & B_{22}
\end{array}\right]=\left[\begin{array}{cc}
\left(2 e^{2}\right)^{-1} & 0 \\
0 & \left(2 e^{2}\right)^{-1}
\end{array}\right],
$$

and

$$
\tilde{A}=\left[\begin{array}{cc}
0 & 1 / 2 \\
2 / 3 & 0
\end{array}\right], \quad \tilde{B}=B=\left[\begin{array}{cc}
\left(2 e^{2}\right)^{-1} & 0 \\
0 & \left(2 e^{2}\right)^{-1}
\end{array}\right]
$$


By choosing $c_{i}=1, \alpha_{i}=4, i=1,2,\|B\|=\left(2 e^{2}\right)^{-1}, \gamma=3,\|\tilde{B}\|=\left(2 e^{2}\right)^{-1}, \tau=1$, $\alpha=2$, then $\left\|R_{i}\left(t, t_{0}\right)\right\| \leq c_{i} e^{-\alpha_{i}\left(t-t_{0}\right)}, i=1,2$, and $\gamma-\|\tilde{B}\| e^{\alpha \tau}-\alpha>0$. Let

$$
D_{k}=\left[\begin{array}{ll}
D_{11 k} & D_{12 k} \\
D_{21 k} & D_{22 k}
\end{array}\right]=\left[\begin{array}{cccc}
e-3 / 2 & 0 & 1 & 1 / 2 \\
1 & 0 & 0 & 1 / 2 \\
0 & 1 & 1 / 2 & 0 \\
0 & -1 & 0 & 0
\end{array}\right]
$$

then $\left\|D_{k}\right\|=\left\|\tilde{D}_{k}\right\|=e-1 / 2$ and if

(1) $t_{k}-t_{k-1} \geq 2>\tau$, then $\left(e^{-2 t} \prod_{j \in \Delta(t)} \max \left\{\left\|\tilde{D}_{j}\right\|, e^{\alpha \tau}\right\}\right) \leq 1$,

(2) $t_{k}-t_{k-1} \geq 2+1 / k$, then $\lim _{t \rightarrow \infty}\left(e^{-2 t} \prod_{j \in \Delta(t)} \max \left(\left\|\tilde{D}_{j}\right\|, e^{\alpha \tau}\right\}\right)=0$,

(3) $t_{k}-t_{k-1} \geq 2+\eta, \eta>0$, then $\left(e^{-t /(2+\eta)} \prod_{j \in \Delta(t)} \max \left\{\left\|\tilde{D}_{j}\right\|, e^{\alpha \tau}\right\}\right) \leq 1$.

Thus we can conclude that system (3.10) is: stable in case (1), asymptotically stable in case (2), and exponentially stable in case (3).

\section{A large-scale impulsive neutral system}

In this section, we consider the large-scale impulsive neutral system

$$
\left\{\begin{aligned}
\frac{d x_{i}(t)}{d t}= & A_{i i}(t) x_{i}(t)+\sum_{j \neq i} A_{i j}(t) x_{j}(t)+\sum_{j=1}^{r} B_{i j}(t) x_{j}(t-\tau) \\
& +\sum_{j=1}^{n} C_{i j}(t) x_{j}^{\prime}(t-\tau), \quad t \in J \backslash I, i=1, \ldots, r, \\
x_{i}\left(t_{k}\right)= & \sum_{j=1}^{r} D_{i j k} x_{j}\left(t_{k}-0\right), \quad t_{k} \in I,
\end{aligned}\right.
$$

where $A_{i j}, B_{i j} \in C\left[J, R^{n_{i} \times n_{j}}\right], D_{i j k} \in R^{n_{i} \times n_{j}}, x_{i}^{T}=\left(x_{1}^{i}, \ldots, x_{n_{i}}^{i}\right) \in R^{n_{i}}, C_{i j} \in$ $C^{1}\left[J, R^{n_{i} \times n_{j}}\right], i, j=1, \ldots, r$, and $\sum_{i=1}^{r} n_{i}=n$.

If $n_{i}=n_{j}=1$ and $D=\left(D_{i j k}\right)=E$ in the system (4.1), where $E$ is an identity matrix, it means that the system does not have an impulse at the point $\tau_{k}$. Then the system becomes

$$
\frac{d x(t)}{d t}=A(t) x(t)+B(t) x(t-\tau)+C(t) x^{\prime}(t-\tau) .
$$

Denote by $R\left(t, t_{0}\right)$ the fundamental matrix of $d x / d t=A(t) x$ and by $x(t)=x\left(t, t_{0}, \phi\right)$ the solution of (4.2) with initial condition $x(t)=\phi(t), t \in\left[t_{0}-\tau, t_{0}\right]$. 


\section{LEMMA 4.1. Assume that}

(1) $\|C(t)\|$ is a decreasing function;

(2) there exist $\gamma \in C\left[R, R^{+}\right], M>0$ such that $\left\|R\left(t, t_{0}\right)\right\| \leq M e^{-\int_{t_{0}}^{\prime} \gamma(s) d s}$;

(3) there exists $l>0$ such that $e^{\int_{t-r}^{\prime} \gamma(s) d s} \leq l$.

Then the solution $x(t)=x\left(t, t_{0}, \phi\right)$ of (4.2) satisfies

$$
\begin{aligned}
\|x(t)\| \leq & {\left[M\left(1+\left\|C\left(t_{0}\right)\right\|\right)+\left\|C\left(t_{0}-\tau\right)\right\| l\right] } \\
& \times\left\|x_{t_{0}}\right\| \exp \left(\int_{t_{0}}^{l}\left[l\left(\frac{1}{\tau}\|C(s)\|+M L(s)\right)-\gamma(s)\right] d s\right),
\end{aligned}
$$

where $L(t)=\|A(t)\|\|C(t)\|+\left\|C^{\prime}(t)\right\|+\|B(t)\|$.

PROOF. By the method of variation of parameters, the solution of (4.2) is given by

$$
\begin{aligned}
x(t)= & R\left(t, t_{0}\right) x\left(t_{0}\right)+\int_{t_{0}}^{t} R(t, s) B(s) x(s-\tau) d s \\
& +\int_{t_{0}}^{t} R(t, s) C(s) x^{\prime}(s-\tau) d s .
\end{aligned}
$$

Integrating by parts, the third term on the right-hand side is

$$
\begin{aligned}
& \int_{t_{0}}^{t} R(t, s) C(s) x^{\prime}(s-\tau) d s \\
&=\int_{t_{0}}^{t} R(t, s) C(s) d x(s-\tau) \\
&=R(t, t) C(t) x(t-\tau)-R\left(t, t_{0}\right) C\left(t_{0}\right) x\left(t_{0}-\tau\right) \\
& \quad-\int_{t_{0}}^{t}\left[R_{s}^{\prime}(t, s) C(s)+R(t, s) C^{\prime}(s)\right] x(s-\tau) d s \\
&=C(t) x(t-\tau)-R\left(t, t_{0}\right) C\left(t_{0}\right) x\left(t_{0}-\tau\right) \\
&-\int_{t_{0}}^{t}\left[-R(t, s) A(s) C(s)+R(t, s) C^{\prime}(s)\right] x(s-\tau) d s
\end{aligned}
$$

since $R(t, t)=E$. So

$$
\begin{aligned}
x(t)= & R\left(t, t_{0}\right) x\left(t_{0}\right)+\int_{t_{0}}^{t} R(t, s) B(s) x(s-\tau) d s+C(t) x(t-\tau) \\
& -R\left(t, t_{0}\right) C\left(t_{0}\right) x\left(t_{0}-\tau\right)+\int_{t_{0}}^{t} R(t, s)\left[A(s) C(s)-C^{\prime}(s)\right] x(s-\tau) d s .
\end{aligned}
$$

Thus

$$
\|x(t)\| \leq\left\|R\left(t, t_{0}\right)\right\|\left\|x\left(t_{0}\right)\right\|+\int_{t_{0}}^{t}\|R(t, s)\|\|B(s)\|\|x(s-\tau)\| d s
$$




$$
\begin{aligned}
& +\|C(t)\|\|x(t-\tau)\|+\left\|R\left(t, t_{0}\right)\right\|\left\|C\left(t_{0}\right)\right\|\left\|x\left(t_{0}-\tau\right)\right\| \\
& +\int_{t_{0}}^{t}\|R(t, s)\|\left[\|A(s)\|\|C(s)\|+\left\|C^{\prime}(s)\right\|\right]\|x(s-\tau)\| d s \\
\leq & M e^{-\int_{t_{0}}^{t} \gamma(s) d s}\left\|x_{t_{0}}\right\|+\int_{t_{0}}^{t} M e^{-\int_{s}^{t} \gamma(\eta) d \eta}\|B(s)\|\|x(s-\tau)\| d s \\
& +\|C(t)\|\|x(t-\tau)\|+M e^{-\int_{t_{0}}^{t} \gamma(s) d s}\left\|C\left(t_{0}\right)\right\|\left\|x_{t_{0}}\right\| \\
& +\int_{t_{0}}^{t} M e^{-\int_{s}^{\prime} \gamma(\eta) d \eta}\left[\|A(s)\|\|C(s)\|+\left\|C^{\prime}(s)\right\|\right]\|x(s-\tau)\| d s \\
= & M e^{-\int_{t_{0}}^{t} \gamma(s) d s}\left\|x_{t_{0}}\right\|\left(1+\left\|C\left(t_{0}\right)\right\|\right)+\|C(t)\|\|x(t-\tau)\| \\
& +\int_{t_{0}}^{t} M e^{-\int_{s}^{t} \gamma(\eta) d \eta}\left[\|A(s)\|\|C(s)\|+\left\|C^{\prime}(s)\right\|+\|B(s)\|\right]\|x(s-\tau)\| d s \\
= & M e^{-\int_{t_{0}}^{t} \gamma(s) d s}\left\|x_{t_{0}}\right\|\left(1+\left\|C\left(t_{0}\right)\right\|\right)+\|C(t)\|\|x(t-\tau)\| \\
& +\int_{t_{0}}^{t} M e^{-\int_{s}^{t} \gamma(\eta) d \eta} L(s)\|x(s-\tau)\| d s .
\end{aligned}
$$

Multiplying both sides by $e^{\int_{10}^{t} \gamma(s) d s}$, we have

$$
\begin{aligned}
e^{\int_{t_{0}}^{t} \gamma(s) d s}\|x(t)\| \leq & M\left\|x_{t_{0}}\right\|\left(1+\left\|C\left(t_{0}\right)\right\|\right)+\|C(t)\| e^{\int_{t_{0}}^{t} \gamma(s) d s}\|x(t-\tau)\| \\
& +\int_{t_{0}}^{t} M e^{\int_{t_{0}}^{s} \gamma(\eta) d \eta} L(s)\|x(s-\tau)\| d s .
\end{aligned}
$$

Let

$$
y(t)=\sup _{t_{0}-\tau \leq s \leq r} e^{\int_{t_{0}}^{s} \gamma(\eta) d \eta}\|x(s)\| .
$$

Then $y(t) \geq e^{\int_{t_{0}}^{t} \gamma(s) d s}\|x(t)\|, t \geq t_{0}-\tau, y(t)$ is a nondecreasing function and

$$
\begin{aligned}
& e^{\int_{t_{0}}^{t} \gamma(s) d s}\|x(t)\| \\
& \leq M\left\|x_{t_{0}}\right\|\left(1+\left\|C\left(t_{0}\right)\right\|\right)+\|C(t)\| e^{\int_{t-\tau}^{t} \gamma(s) d s} e^{\int_{t_{0}}^{t-\tau} \gamma(s) d s}\|x(t-\tau)\| \\
&+\int_{t_{0}}^{t} M e^{\int_{s-z}^{s} \gamma(\eta) d \eta} e^{\int_{t_{0}}^{s-\tau} \gamma(\eta) d \eta} L(s)\|x(s-\tau)\| d s \\
& \leq M\left\|x_{t_{0}}\right\|\left(1+\left\|C\left(t_{0}\right)\right\|\right)+\|C(t)\| l y(t-\tau)+\int_{t_{0}}^{t} M l L(s) y(s-\tau) d s \\
& \leq M\left\|x_{t_{0}}\right\|\left(1+\left\|C\left(t_{0}\right)\right\|\right)+\frac{l}{\tau} \int_{t-\tau}^{t}\|C(s)\| y(s) d s \\
&+\int_{t_{0}}^{t} M l L(s) y(s-\tau) d s,
\end{aligned}
$$


in view of the fact that $\|C(t)\|$ is nonincreasing and $y(t)$ is nondecreasing. Thus

$$
\begin{aligned}
e^{f_{t_{0}}^{t} y(s) d s}\|x(t)\| \leq & M\left\|x_{t_{0}}\right\|\left(1+\left\|C\left(t_{0}\right)\right\|\right)+\int_{t_{0}-\tau}^{t_{0}} \frac{l}{\tau}\|C(s)\| y(s) d s \\
& +\int_{t_{0}}^{t} \frac{l}{\tau}\|C(s)\| y(s) d s+\int_{t_{0}}^{t} M L L(s) y(s) d s \\
\leq & M\left\|x_{t_{0}}\right\|\left(1+\left\|C\left(t_{0}\right)\right\|\right)+l\left\|C\left(t_{0}-\tau\right)\right\|\left\|x_{t_{0}}\right\| \\
& +\int_{t_{0}}^{t} \frac{l}{\tau}\|C(s)\| y(s) d s+\int_{t_{0}}^{t} M l L(s) y(s) d s \\
\leq & \left\|x_{t_{0}}\right\|\left[\left(1+\left\|C\left(t_{0}\right)\right\|\right) M+\left\|C\left(t_{0}-\tau\right)\right\| l\right] \\
& +\int_{t_{0}}^{t} l\left[\frac{1}{\tau}\|C(s)\|+M L(s)\right] y(s) d s .
\end{aligned}
$$

The right-hand side of the last inequality is nondecreasing and it yields that

$$
\begin{aligned}
y(t) \leq & \left\|x_{t_{0}}\right\|\left[\left(1+\left\|C\left(t_{0}\right)\right\|\right) M+\left\|C\left(t_{0}-\tau\right)\right\| l\right] \\
& +\int_{t_{0}}^{t} l\left[\frac{1}{\tau}\|C(s)\|+M L(s)\right] y(s) d s .
\end{aligned}
$$

The Gronwall-Bellman inequality implies

$$
y(t) \leq\left\|x_{t_{0}}\right\|\left[\left(1+\left\|C\left(t_{0}\right)\right\|\right) M+\left\|C\left(t_{0}-\tau\right)\right\| l\right] e^{\int_{t_{0}}^{\prime}\left[\frac{1}{\tau}\|C(s)\|+M L(s)\right) d s}
$$

and so (4.3) holds. The proof is complete.

LEMMA 4.2. Assume that conditions (2)-(3) of Lemma 4.1 hold and there exists $\tilde{C}>0$ such that $\|C(t)\| \leq \tilde{C}<1 / l$. Then the solution $x(t)$ of (4.2) satisfies

$$
\|x(t)\| \leq \frac{M\left(1+\left\|C\left(t_{0}\right)\right\|\right)}{1-\tilde{C} l}\left\|x_{t_{0}}\right\| \exp \left(\int_{t_{0}}^{t}\left[\frac{M l L(s)}{1-\tilde{C} l}-\gamma(s)\right] d s\right),
$$

where $L(t)=\|A(t)\|\|C(t)\|+\left\|C^{\prime}(t)\right\|+\|B(t)\|$.

PROOF. From the first part of the proof of Lemma 4.1, it follows that

$$
\begin{aligned}
e^{\int_{t_{0}}^{t} \gamma(s) d s}\|x(t)\| \leq & M\left\|x_{t_{0}}\right\|\left(1+\left\|C\left(t_{0}\right)\right\|\right) \\
& +\|C(t)\| e^{\int_{t-\tau}^{\prime} \gamma(s) d s} e^{f_{t_{0}}^{t-\tau} \gamma(s) d s}\|x(t-\tau)\| \\
& +\int_{t_{0}}^{t} M e^{\int_{s-r}^{s} \gamma(\eta) d \eta} e^{\int_{t_{0}}^{s-r} \gamma(\eta) d \eta} L(s)\|x(s-\tau)\| d s \\
\leq & M\left\|x_{t_{0}}\right\|\left(1+\left\|C\left(t_{0}\right)\right\|\right)+\|C(t)\| l y(t-\tau)
\end{aligned}
$$




$$
\begin{aligned}
& +\int_{t_{0}}^{t} M l L(s) y(s-\tau) d s \\
\leq & M\left\|x_{t_{0}}\right\|\left(1+\left\|C\left(t_{0}\right)\right\|\right)+\tilde{C} l y(t)+\int_{t_{0}}^{t} M l L(s) y(s) d s,
\end{aligned}
$$

where $y(t) \geq e^{\int_{t_{0}}^{\prime} \gamma(s) d s}\|x(t)\|, t \geq t_{0}-\tau$, is a nondecreasing function. Furthermore, since the right-hand side of the last inequality is nondecreasing, it follows that

$$
y(t) \leq M\left\|x_{t_{0}}\right\|\left(1+\left\|C\left(t_{0}\right)\right\|\right)+\tilde{C} l y(t)+\int_{t_{0}}^{t} M l L(s) y(s) d s,
$$

thus

$$
y(t) \leq \frac{M\left\|x_{t_{0}}\right\|\left(1+\left\|C\left(t_{0}\right)\right\|\right)}{1-\tilde{C} l}+\frac{1}{1-\tilde{C} l} \int_{t_{0}}^{t} M l L(s) y(s) d s .
$$

The Gronwall-Bellman inequality implies

$$
y(t) \leq \frac{M\left(1+\left\|C\left(t_{0}\right)\right\|\right)}{1-\tilde{C} l}\left\|x_{t_{0}}\right\| e^{\int_{t_{0}}^{t} \frac{M(L(s)}{1-\tilde{C} l} d s}
$$

and so (4.5) holds. The proof is complete.

THEOREM 4.3. Assume that the conditions of Lemma 4.1 hold and $x(t)=x\left(t, t_{0}, \phi\right)$ is the solution of the system

$$
\begin{cases}d x / d t=A(t) x(t)+B(t) x(t-\tau)+C(t) x^{\prime}(t-\tau), & t \neq t_{k}, \\ x\left(t_{k}\right)=D_{k} x\left(t_{k}-0\right), & \theta \in\left[t_{0}-\tau, t_{0}\right] . \\ x(\theta)=\phi(\theta), & \end{cases}
$$

Then for $t_{k} \leq t<t_{k+1}$,

$$
\|x(t)\| \leq\left\|x_{s_{0}}\right\| M_{0} \prod_{I \leq j \leq k} M_{j} \max \left\{\sup _{t_{j}-\tau \leq t<t_{j}} e^{\int_{i j}^{t} \alpha(s) d s},\left\|D_{j}\right\|\right\} e^{f_{t_{0}}^{\prime} \alpha(s) d s},
$$

where $\alpha(t)=l\left(\frac{1}{r}\|C(s)\|+M L(s)\right)-\gamma(s), M_{k}=M\left(1+\left\|C\left(t_{k}\right)\right\|\right)+\left\|C\left(t_{k}-\tau\right)\right\| l$, $L(t)=\|A(t)\|\|C(t)\|+\left\|C^{\prime}(t)\right\|+\|B(t)\|$ and $l \geq e^{\int_{t-8}^{t} \gamma(s) d s}, t \geq t_{0}$.

PROOF. From Lemma 4.1, for any $k=1, \ldots$, we have

$$
\|x(t)\| \leq M_{k}\left\|x_{t_{k}}\right\| e^{\int_{t_{k}}^{\prime} \alpha(s) d s}, \quad t \in\left[t_{k}, t_{k+1}\right)
$$

Since $x\left(t_{k}\right)=D_{k} x\left(t_{k}-0\right)$, it follows that

$$
\left\|x_{t_{k}}\right\|=\sup _{t_{t}-t \leq t \leq t_{k}}\|x(t)\|=\max \left\{\sup _{t_{k}-t \leq t<t_{k}}\|x(t)\|,\left\|x\left(t_{k}\right)\right\|\right\}
$$




$$
\begin{aligned}
& \leq \max \left\{\sup _{t_{t}-r \leq t<t_{k}}\|x(t)\|,\left\|D_{k}\right\|\left\|x\left(t_{k}-0\right)\right\|\right\} \\
& \leq \max \left\{\sup _{t_{k}-\tau \leq t<t_{k}} M_{k-1}\left\|x_{t_{k-1}}\right\| e^{\int_{t_{k-1}}^{t} \alpha(s) d s}, M_{k-1}\left\|D_{k}\right\|\left\|x_{t_{k-1}}\right\| e^{\int_{t_{k-1}}^{t_{k}} \alpha(s) d s}\right\}
\end{aligned}
$$

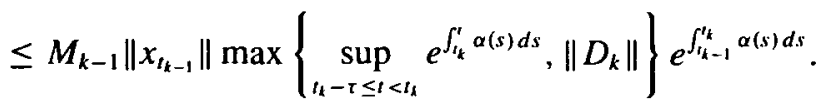

Using a similar argument, we have that

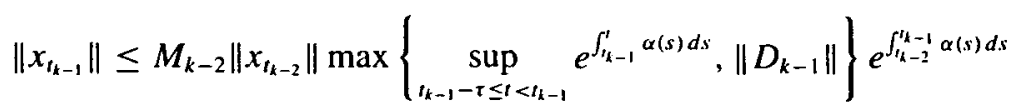

and so on. Thus we get, for $t_{k} \leq t<t_{k+1}$,

$$
\begin{aligned}
\|x(t)\| & \leq M_{k}\left\|x_{t_{k}}\right\| e^{\int_{t_{k}}^{t} \alpha(s) d s} \\
& \leq M_{k} M_{k-1}\left\|x_{t_{k-1}}\right\| \max \left\{\sup _{t_{t}-\tau \leq t<t_{k}} e^{\int_{t_{k}}^{t} \alpha(s) d s},\left\|D_{k}\right\|\right\} e^{\int_{t_{k-1}}^{t_{k}} \alpha(s) d s} e^{\int_{t_{k}}^{t} \alpha(s) d s} \\
& =M_{k} M_{k-1}\left\|x_{t_{k-1}}\right\| \max \left\{\sup _{t_{k}-\tau \leq t<t_{k}} e^{\int_{t_{k}}^{t} \alpha(s) d s},\left\|D_{k}\right\|\right\} e^{\int_{t_{k}-1}^{t} \alpha(s) d s} \\
& \leq \cdots \leq\left\|x_{t_{0}}\right\| M_{0} \prod_{1 \leq j \leq k} M_{j} \max \left\{\sup _{t_{j}-r \leq t<t_{j}} e^{\int_{t_{j}}^{\prime} \alpha(s) d s},\left\|D_{j}\right\|\right\} e^{\int_{t_{0}}^{t} \alpha(s) d s} .
\end{aligned}
$$

The proof is complete.

THEOREM 4.4. Assume that the conditions of Lemma 4.2 hold and that $x(t)$ is the solution of (4.6). Then for $t_{k} \leq t<t_{k+1}$,

$$
\|x(t)\| \leq\left\|x_{t_{0}}\right\| M_{0} \prod_{i \leq j \leq k} M_{j} \max \left\{\sup _{t_{j}-t \leq t<t_{j}} e^{\int_{t_{j}}^{t} \alpha(s) d s},\left\|D_{j}\right\|\right\} e^{\int_{t_{0}}^{t} \alpha(s) d s},
$$

where $\alpha(t)=M l L(s) /(1-\tilde{C} l)-\gamma(s), M_{k}=M\left(1+\left\|C\left(t_{k}\right)\right\|\right) /(1-\tilde{C} l), L(t)=$ $\|A(t)\|\|C(t)\|+\left\|C^{\prime}(t)\right\|+\|B(t)\|$ and $l \geq \int_{t-t}^{t} \gamma(s) d s$.

PROOF. The proof is similar to that of Theorem 4.3, and we therefore omit it.

It is easy to obtain the following result from Theorems 4.3 and 4.4.

COROLlaRY 4.5. Assume that the conditions of Theorems 4.3 or 4.4 hold. Then

(1) $\lim \sup _{t \rightarrow \infty}\left(e^{\int_{t_{0}}^{\prime} \alpha(s) d s} \prod_{j \in \Delta(t)} M_{j} \max \left\{\left\|D_{j}\right\|, \sup _{t_{j}-\tau \leq t \leq t_{j}} e^{\int_{t j}^{t} \alpha(x) d s}\right\}\right)<\infty$ implies system (4.6) is stable; 
(2) $\lim \sup _{t \rightarrow \infty}\left(e^{\int_{t_{0}}^{\prime} \alpha(s) d s} \prod_{j \in \Delta(t)} M_{j} \max \left\{\left\|D_{j}\right\|, \sup _{t_{j}-\tau \leq t \leq t_{j}} e^{\int_{t_{j}}^{\prime} \alpha(s) d s}\right\}\right)=0$ implies system (4.6) is asymptotically stable,

where $M_{j}$ and $\alpha(t)$ are as in Theorem 4.3 or Theorem 4.4 respectively according to which conditions hold.

COROLlaRY 4.6. Assume that the conditions of Theorems 4.3 or 4.4 hold and $\alpha(t)=-\alpha=$ constant. Furthermore, there exist a constant $\eta>\tau$ and $M>0$ such that $t_{k}-t_{k-1}=\eta$ and $M_{j} \max \left\{e^{\alpha \tau},\left\|D_{j}\right\|\right\} \leq M$. Then

(1) $M \leq e^{\alpha \eta}$ implies system (4.6) is stable;

(2) $M<e^{\alpha \eta}$ implies system (4.6) is exponentially stable.

Next, we will consider a large-scale impulsive neutral system (4.1). Assume that $R_{i i}(t, s)$ satisfies $R_{i i}(t, t)=E$ and

$$
\frac{\partial R_{i i}(t, s)}{\partial t}=A_{i i}(t) R_{i i}(t, s), \quad t \in J \backslash I, i=1, \ldots, r .
$$

THEOREM 4.7. Assume that

(1) there exist a scalar function $\alpha \in C[J, R]$ and constant $M_{i} \geq 1$ such that

$$
\left\|R_{i i}\left(t, t_{0}\right)\right\| \leq M_{i} e^{-\int_{i_{0}}^{t} \alpha(\xi) d \xi}
$$

(2) $\left\|C_{i j}(t)\right\|$ are nonincreasing functions;

(3) there exist $N>0, l>0$ and a scalar function $\beta \in C[l, R]$ such that $l \geq \sup _{t \geq t_{0}} e^{\int_{t \rightarrow \mathrm{r}}^{\prime} \alpha(s) d s}$ and the fundamental matrix solution $Q\left(t, t_{0}\right)$ of the ordinary differential equations

$$
\frac{d \eta_{i}}{d t}=\sum_{j=1}^{r} \nu_{i j}(t) \eta_{j}, \quad i=1, \ldots, r,
$$

satisfies $\left\|Q\left(t, t_{0}\right)\right\| \leq N e^{\int_{t_{0}}^{\prime} \beta(s) d s}$, where

$$
\begin{aligned}
v_{i j} & =\left(1-\delta_{i j}\right) M_{i}\left\|A_{i j}(s)\right\|+M_{i} l L(s)+\frac{l}{\tau}\left\|C_{i j}(s)\right\| \text { and } \\
L(t) & =\left\|B_{i j}(t)\right\|+\left\|C_{i j}^{\prime}(t)\right\|+\left\|A_{i i}(t)\right\|\left\|C_{i j}(t)\right\| .
\end{aligned}
$$

If $M=\max _{i \leq i \leq r} M_{i}, \tilde{M}_{k}=N\left[M+(M+l)\left\|C_{i_{k}}\right\|\right]$, then

(1) system (4.1) is stable if

$$
\limsup _{t \rightarrow \infty} \frac{\prod_{j \in \Delta(t)} \tilde{M}_{j} \max \left\{\left\|D_{j}\right\|, \sup _{t_{j}-r \leq s<t_{j}} e^{\int_{t_{j}}^{\prime}(\beta(s)-\alpha(s)) d s}\right\}}{e^{\int_{t_{0}}^{\prime}(\alpha(s)-\beta(s)) d s}}<\infty
$$


(2) system (4.1) is asymptotically stable if

$$
\limsup _{t \rightarrow \infty} \frac{\prod_{j \in \Delta(t)} \tilde{M}_{j} \max \left\{\left\|D_{j}\right\|, \sup _{t_{j}-\tau \leq t<t_{j}} e^{\int_{t_{i}}^{\prime}(\beta(s)-\alpha(s)) d s}\right\}}{e^{\int_{t_{0}}^{\prime}(\alpha(s)-\beta(s)) d s}}=0 ;
$$

(3) if there exists a positive number $\mu$ such that

$$
\limsup _{t \rightarrow \infty} \frac{\prod_{j \in \Delta(t)} \tilde{M}_{j} \max \left\{\left\|D_{j}\right\|, \sup _{t_{j}-\tau \leq t<t j} e^{\int_{t_{j}}^{t}(\beta(s)-\alpha(s)) d s}\right\}}{e^{\int_{t_{0}}^{\prime}(\alpha(s)-\beta(s)+\mu) d s}}=0
$$

then system (4.1) is exponentially stable.

PROOF. Using the method of variation of parameters, the solution of system (4.1) can be written as

$$
\begin{aligned}
x_{i}(t)= & R_{i i}\left(t, t_{0}\right) x_{i}\left(t_{0}\right)+\int_{t_{0}}^{t} R_{i i}(t, s) \sum_{j \neq i} A_{i j}(s) x_{j}(s) d s \\
& +\int_{t_{0}}^{t} R_{i i}(t, s) \sum_{j=1}^{r} B_{i j}(s) x_{j}(s-\tau) d s+\int_{t_{0}}^{t} R_{i i}(t, s) \sum_{j=1}^{r} C_{i j}(s) x_{j}^{\prime}(s-\tau) d s .
\end{aligned}
$$

Since

$$
\begin{aligned}
\int_{t_{0}}^{t} R_{i i}(t, s) & \sum_{j=1}^{r} C_{i j}(s) x_{j}^{\prime}(s-\tau) d s \\
= & \sum_{j=1}^{r} C_{i j}(t) x_{j}(t-\tau)-R_{i i}\left(t, t_{0}\right) \sum_{j=1}^{r} C_{i j}\left(t_{0}\right) x_{j}\left(t_{0}-\tau\right) \\
& -\int_{t_{0}}^{t} R_{i i}(t, s) \sum_{j=1}^{r} C_{i j}^{\prime}(s) x_{j}(s-\tau) d s \\
& +\int_{t_{0}}^{t} R_{i i}(t, s) A_{i i}(s) \sum_{j=1}^{r} C_{i j}(s) x_{j}(s-\tau) d s
\end{aligned}
$$

then

$$
\begin{aligned}
x_{i}(t)= & R_{i i}\left(t, t_{0}\right) x_{i}\left(t_{0}\right)+\int_{t_{0}}^{t} R_{i i}(t, s) \sum_{j \neq i} A_{i j}(s) x_{j}(s) d s \\
& +\int_{t_{0}}^{t} R_{i i}(t, s) \sum_{j=1}^{r} B_{i j}(s) x_{j}(s-\tau) d s
\end{aligned}
$$




$$
\begin{aligned}
& +\sum_{j=1}^{r} C_{i j}(t) x_{j}(t-\tau)-R_{i i}\left(t, t_{0}\right) \sum_{j=1}^{r} C_{i j}\left(t_{0}\right) x_{j}\left(t_{0}-\tau\right) \\
& -\int_{t_{0}}^{t} R_{i i}(t, s) \sum_{j=1}^{r} C_{i j}^{\prime}(s) x_{j}(s-\tau) d s \\
& +\int_{t_{0}}^{t} R_{i i}(t, s) A_{i i}(s) \sum_{j=1}^{r} C_{i j}(s) x_{j}(s-\tau) d s .
\end{aligned}
$$

Thus we have

$$
\begin{aligned}
\left\|x_{i}(t)\right\| \leq & M_{i} e^{-\int_{10}^{\prime} \alpha(s) d s}\left\|x_{i t_{0}}\right\| \\
& +\sum_{j=1}^{r} \int_{t_{0}}^{t}\left(1-\delta_{i j}\right) M_{i}\left\|A_{i j}(s)\right\| e^{-\int_{s}^{\prime} \alpha(\xi) d \xi}\left\|\dot{x}_{j}(s)\right\| d s \\
& +\sum_{j=1}^{r} \int_{t_{0}}^{t} M_{i}\left\|B_{i j}(s)\right\| e^{-\int_{s}^{\prime} \alpha(\xi) d \xi}\left\|x_{j}(s-\tau)\right\| d s \\
& +\sum_{j=1}^{r}\left\|C_{i j}(t)\right\|\left\|x_{j}(t-\tau)\right\|+M_{i} e^{-\int_{i_{0}}^{\prime} \alpha(s) d s} \sum_{j=1}^{r}\left\|C_{i j}\left(t_{0}\right)\right\|\left\|x_{j}\left(t_{0}-\tau\right)\right\| \\
& +\int_{t_{0}}^{t} M_{i} e^{-\int_{s}^{t} \alpha(\xi) d \xi} \sum_{j=1}^{r}\left\|C_{i j}^{\prime}(s)\right\| x_{j}(s-\tau) \| d s \\
& +\int_{t_{0}}^{t} M_{i} e^{-\int_{t}^{\prime} \alpha(\xi) d \xi}\left\|A_{i i}(s)\right\| \sum_{j=1}^{r}\left\|C_{i j}(s)\right\|\left\|x_{j}(s-\tau)\right\| d s
\end{aligned}
$$

and

$$
\begin{aligned}
\left\|x_{i}(t)\right\| e^{\int_{t_{0}}^{\prime} \alpha(s) d s} \leq & M_{i}\left\|x_{i t_{0}}\right\|+\sum_{j=1}^{r} \int_{t_{0}}^{t}\left(1-\delta_{i j}\right) M_{i}\left\|A_{i j}(s)\right\| e^{\int_{t_{0}}^{s} \alpha(\xi) d \xi}\left\|x_{j}(s)\right\| d s \\
& +\sum_{j=1}^{r} \int_{t_{0}}^{t} M_{i}\left\|B_{i j}(s)\right\| e^{\int_{t_{0}}^{s} \alpha(\xi) d \xi}\left\|x_{j}(s-\tau)\right\| d s \\
& +e^{\int_{t_{0}}^{\prime} \alpha(s) d s} \sum_{j=1}^{r}\left\|C_{i j}(t)\right\|\left\|x_{j}(t-\tau)\right\|+M_{i} \sum_{j=1}^{r}\left\|C_{i j}\left(t_{0}\right)\right\|\left\|x_{j t_{0}}\right\| \\
& +\int_{t_{0}}^{r} M_{i} e^{\int_{t_{0}}^{s} \alpha(\xi) d \xi} \sum_{j=1}^{r}\left\|C_{i j}^{\prime}(s)\right\| x_{j}(s-\tau) \| d s \\
& +\int_{t_{0}}^{t} M_{i} e^{\int_{t_{0}}^{s} \alpha(\xi) d \xi}\left\|A_{i j}(s)\right\| \sum_{j=1}^{r}\left\|C_{i j}(s)\right\|\left\|x_{j}(s-\tau)\right\| d s
\end{aligned}
$$




$$
\begin{aligned}
= & M_{i}\left\|x_{i t_{0}}\right\|+M_{i} \sum_{j=1}^{r}\left\|C_{i j}\left(t_{0}\right)\right\|\left\|x_{j t_{0}}\right\| \\
& +\sum_{j=1}^{r} \int_{t_{0}}^{t}\left(1-\delta_{i j}\right) M_{i}\left\|A_{i j}(s)\right\| e^{\int_{\iota_{0}}^{s} \alpha(\xi) d \xi}\left\|x_{j}(s)\right\| d s \\
& +\sum_{j=1}^{r} \int_{t_{0}}^{t} M_{i} e^{\int_{t_{0}}^{s} \alpha(\xi) d \xi}\left[\left\|B_{i j}(s)\right\|+\left\|C_{i j}^{\prime}(s)\right\|\right. \\
& \left.+\left\|A_{i i}(s)\right\|\left\|C_{i j}(s)\right\|\right]\left\|x_{j}(s-\tau)\right\| d s \\
& +e^{\int_{t_{0}}^{s} \alpha(s) d s} \sum_{j=1}^{r}\left\|C_{i j}(t)\right\|\left\|x_{j}(t-\tau)\right\| .
\end{aligned}
$$

Let $y_{i}(t)=\max _{t_{0}-\tau \leq \xi \leq t}\left\|x_{i}(\xi)\right\| e^{\int_{t_{0}}^{\xi} \alpha(s) d s}$, then $y_{i}(t)$ is a nondecreasing function, $i=1, \ldots, r$. As $y_{i}(t)$ is nondecreasing, $\left\|C_{i j}(t)\right\|$ is nonincreasing, and because $l \geq \sup _{t \geq t_{0}} e^{\int_{t-r}^{t} \alpha(s) d s}$, we have

$$
\begin{aligned}
\sum_{j=1}^{r} \| & C_{i j}(t)\|\| x_{j}(t-\tau) \| e^{\int_{t_{0}}^{t} \alpha(s) d s} \\
& \leq \sum_{j=1}^{r}\left\|C_{i j}(t)\right\| y_{j}(t-\tau) e^{\int_{t-\tau}^{t} \alpha(s) d s} \\
& \leq \sum_{j=1}^{r} \frac{l}{\tau} \int_{t-\tau}^{t}\left\|C_{i j}(t)\right\| y_{j}(t-\tau) d s \leq \sum_{j=1}^{r} \frac{l}{\tau} \int_{t-\tau}^{r}\left\|C_{i j}(s)\right\| y_{j}(s) d s \\
& \leq \sum_{j=1}^{r} \frac{l}{\tau} \int_{t_{0}}^{t}\left\|C_{i j}(s)\right\| y_{j}(s) d s+\sum_{j=1}^{r} \frac{l}{\tau} \int_{t_{0}-\tau}^{t_{0}}\left\|C_{i j}(s)\right\| y_{j}(s) d s \\
& \leq \sum_{j=1}^{r} \frac{l}{\tau} \int_{t_{0}}^{t}\left\|C_{i j}(s)\right\| y_{j}(s) d s+\sum_{j=1}^{r} l\left\|C_{i j}\left(t_{0}-\tau\right)\right\|\left\|x_{j t_{0}}\right\| .
\end{aligned}
$$

Thus

$$
\begin{aligned}
\left\|x_{i}(t)\right\| e^{f_{t_{0}}^{t} \alpha(s) d s} & \leq M_{i}\left\|x_{i t_{0}}\right\|+M_{i} \sum_{j=1}^{r}\left\|C_{i j}\left(t_{0}\right)\right\|\left\|x_{j t_{0}}\right\| \\
& +\sum_{j=1}^{r} \int_{t_{0}}^{t}\left(1-\delta_{i j}\right) M_{i}\left\|A_{i j}(s)\right\| y_{j}(s) d s+\sum_{j=1}^{r} \int_{t_{0}}^{t} M_{i} l L(s) \| y_{j}(s) d s \\
& +\sum_{j=1}^{r} \frac{l}{\tau} \int_{t_{0}}^{t}\left\|C_{i j}(s)\right\| y_{j}(s) d s+\sum_{j=1}^{r} l\left\|C_{i j}\left(t_{0}-\tau\right)\right\|\left\|x_{j t_{0}}\right\|
\end{aligned}
$$




$$
\begin{aligned}
= & \left.M_{i}\left\|x_{i t_{0}}\right\|+\sum_{j=1}^{r}\left[M_{i}\left\|C_{i j}\left(t_{0}\right)\right\|+l\left\|C_{i j}\left(t_{0}-\tau\right)\right\|\right)\right]\left\|x_{j t_{0}}\right\| \\
& +\sum_{j=1}^{r} \int_{t_{0}}^{t}\left[\left(1-\delta_{i j}\right) M_{i}\left\|A_{i j}(s)\right\|+M_{i} l L(s)+\frac{l}{\tau}\left\|C_{i j}(s)\right\|\right] y_{j}(s) d s .
\end{aligned}
$$

The right-hand side of the last inequality is increasing and hence

$$
\begin{aligned}
y_{i}(t) \leq & M_{i}\left\|x_{i t_{0}}\right\|+\sum_{j=1}^{r}\left[M_{i}\left\|C_{i j}\left(t_{0}\right)\right\|+l\left\|C_{i j}\left(t_{0}-\tau\right)\right\|\right]\left\|x_{j t_{0}}\right\| \\
& +\sum_{j=1}^{r} \int_{t_{0}}^{t}\left[\left(1-\delta_{i j}\right) M_{i}\left\|A_{i j}(s)\right\|+M_{i} l L(s)+\frac{l}{\tau}\left\|C_{i j}(s)\right\|\right] y_{j}(s) d s \\
\leq & M_{i}\left\|x_{i t_{0}}\right\|+\left(M_{i}+l\right) \sum_{j=1}^{r}\left\|C_{i j t_{0}}\right\|\left\|x_{j t_{0}}\right\| \\
& +\sum_{j=1}^{r} \int_{t_{0}}^{t}\left[\left(1-\delta_{i j}\right) M_{i}\left\|A_{i j}(s)\right\|+M_{i} l L(s)+\frac{l}{\tau}\left\|C_{i j}(s)\right\|\right] y_{j}(s) d s \\
\leq & M\left\|x_{i t_{0}}\right\|+(M+l) \sum_{j=1}^{r}\left\|C_{i j t_{0}}\right\|\left\|x_{j t_{0}}\right\| \\
& +\sum_{j=1}^{r} \int_{t_{0}}^{t}\left[\left(1-\delta_{i j}\right) M_{i}\left\|A_{i j}(s)\right\|+M_{i} l L(s)+\frac{l}{\tau}\left\|C_{i j}(s)\right\|\right] y_{j}(s) d s \\
= & \bar{M}_{i}+\sum_{j=1}^{r} \int_{t_{0}}^{t} v_{i j}(s) y_{j}(s) d s .
\end{aligned}
$$

Let $P_{i}(t)=\bar{M}_{i}+\sum_{j=1}^{r} \int_{t_{0}}^{t} \nu_{i j}(s) y_{j}(s) d s$. Then $P_{i}(t) \geq y_{i}(t), P_{i}\left(t_{0}\right)=\bar{M}_{i}$ and

$$
P_{i}^{\prime}(t)=\sum_{j=1}^{r} v_{i j}(t) y_{j}(t) \leq \sum_{j=1}^{r} v_{i j}(t) P_{j}(t)
$$

Consider the system

$$
\left\{\begin{array}{l}
d \xi_{i} / d t=\sum_{j=1}^{r} v_{i j}(s) \xi_{j}(s), \quad t \in\left[t_{0}, t_{1}\right) \\
\xi\left(t_{0}\right)=\bar{M}
\end{array}\right.
$$

where $\xi(s)=\operatorname{col}\left(\xi_{1}(s), \ldots, \xi_{r}(s)\right)$ and $\bar{M}=\operatorname{col}\left(\bar{M}_{1}, \ldots, \bar{M}_{r}\right)$. It is obvious that

$$
\xi_{i}(t) \geq P_{i}(t) \geq y_{i}(t) \geq\left\|x_{i}(t)\right\| e^{\int_{10}^{\prime} \alpha(s) d s}, \quad t \in\left[t_{0}-\tau, t_{1}\right)
$$


and thus $\|\xi(t)\| \geq\|x(t)\| e^{\int_{t_{0}}^{\prime} \alpha(s) d s}$. Furthermore, from condition (3), it follows that the solution $\xi(t)$ of (4.8) satisfies $\|\xi(t)\| \leq N\left\|\xi\left(t_{0}\right)\right\| e^{\int_{t_{0}}^{t} \beta(s) d s}$ on $\left[t_{0}, t_{1}\right)$ and

$$
\begin{aligned}
\left\|\xi\left(t_{0}\right)\right\| & =\|\bar{M}\|=\sum_{i=1}^{r} \bar{M}_{i}=\sum_{i=1}^{r}\left[M\left\|x_{i t_{0}}\right\|+(M+l) \sum_{j=1}^{r}\left\|C_{i j t_{0}}\right\|\left\|x_{j t_{0}}\right\|\right] \\
& =M\left\|x_{t_{0}}\right\|+(M+l) \sum_{i=1}^{r}\left[\sum_{j=1}^{r}\left\|C_{i j t_{0}}\right\|\right]\left\|x_{j t_{0}}\right\| \\
& \leq M\left\|x_{t_{0}}\right\|+(M+l)\left\|C_{t_{0}}\right\|\left\|x_{t_{0}}\right\| .
\end{aligned}
$$

Thus

$$
\begin{aligned}
& \|\xi(t)\| \leq N\left[M+(M+l)\left\|C_{t_{0}}\right\|\right]\left\|x_{t_{0}}\right\| e^{\int_{t_{0}}^{t} \beta(s) d s} \quad \text { and } \\
& \|x(t)\| \leq\|\xi(t)\| e^{-\int_{t_{0}}^{\prime} \alpha(s) d s} \leq N\left[M+(M+l)\left\|C_{t_{0}}\right\|\right]\left\|x_{t_{0}}\right\| e^{\int_{t_{0}}^{t}(\beta(s)-\alpha(s)] d s} .
\end{aligned}
$$

Using the same argument, it is easy to get that

$$
x(t) \leq N\left[M+(M+l)\left\|C_{t_{k}}\right\|\right]\left\|x_{t_{k}}\right\| e^{\int_{l_{k}}^{t}[\beta(s)-\alpha(s)] d s}, \quad t \in\left[t_{k-1}, t_{k}\right) .
$$

Furthermore,

$$
\begin{aligned}
& \left\|x_{t_{k}}\right\|=\sup _{t_{k}-\tau \leq t \leq t_{k}}\|x(t)\|=\max \left\{\left\|x\left(t_{k}\right)\right\|, \sup _{t_{k}-\tau \leq t<t_{k}}\|x(t)\|\right\} \\
& \leq \max \left\{\left\|D_{k}\right\|\left\|x\left(t_{k}-0\right)\right\|, \sup _{t_{k}-\tau \leq t<t_{k}} \tilde{M}_{k-1}\|x(t)\|\right\}
\end{aligned}
$$

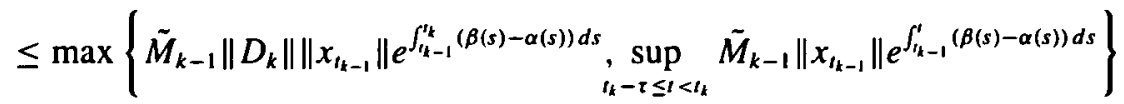

$$
\begin{aligned}
& \leq \max \left\{\left\|D_{k}\right\|, \sup _{t_{k}-\tau \leq i<i_{k}} e^{\int_{i_{k}}^{\prime}(\beta(s)-\alpha(s)) d s}\right\} \tilde{M}_{k-1}\left\|x_{t_{k-1}}\right\| e^{\int_{k-1}^{k}(\beta(s)-\alpha(s)) d s} \text {. }
\end{aligned}
$$

Thus when $t \in\left[t_{k}, t_{k+1}\right)$,

$$
\begin{aligned}
\|x(t)\| & \leq \tilde{M}_{k}\left\|x_{t_{t}}\right\| e^{\int_{t_{k}}^{t}[\beta(s)-\alpha(s)] d s} \\
& \leq \tilde{M}_{k} \tilde{M}_{k-1} \max \left\{\left\|D_{k}\right\|, \sup _{t_{k}-\tau \leq t<l_{k}} e^{\int_{t_{k}}^{\prime}(\beta(s)-\alpha(s)) d s}\right\}\left\|x_{t_{t-1}}\right\| e^{\int_{t_{k-1}}^{\prime}(\beta(s)-\alpha(s)) d s} \\
& \leq \cdots \leq \tilde{M}_{0} \prod_{j \in \Delta(t)} \tilde{M}_{j} \max \left\{\left\|D_{j}\right\|_{t_{j}-\tau \leq t<t_{j}} e^{\int_{t_{j}}(\beta(s)-\alpha(s)) d s}\right\}\left\|x_{t_{0}}\right\| e^{\int_{t_{0}}^{t}(\beta(s)-\alpha(s)) d s} .
\end{aligned}
$$

The inequality implies all of the results of Theorem 4.7. The proof is complete. 
THEOREM 4.8. Assume that conditions (1)-(2) of Theorem 4.7 hold. Let

$$
\begin{gathered}
\tilde{C}(t)=\left(\left\|C_{i j}(t)\right\|\right), \quad \tilde{A}(t)=\left(\left(1-\delta_{i j}\right)\left\|A_{i j(t)}\right\|\right), \quad L(t)=\left(L_{i j}(t)\right), \\
M=\max _{1 \leq i \leq r} M_{i}, \quad \tilde{M}_{k}=\frac{M\left(1+\left\|\tilde{C}\left(t_{k}\right)\right\|\right)}{1-\left\|\tilde{C}\left(t_{k}\right)\right\|}, \quad \beta(t)=\frac{M(\|\tilde{A}(t)\|+l\|L(t)\|)}{1-\left\|\tilde{C}\left(t_{0}\right)\right\|}, \\
l=\sup _{t \geq t_{0}} e^{\int_{t-}^{\prime} \alpha(s) d s}<+\infty \quad \text { and } \quad L_{i j}(t)=\left\|B_{i j}(t)\right\|+\left\|C_{i j}^{\prime}(t)\right\|+\left\|A_{i i}(t)\right\|\left\|C_{i j}(t)\right\| .
\end{gathered}
$$

\section{Then}

(1) system (4.1) is stable if

$$
\limsup _{t \rightarrow \infty} \frac{\prod_{j \in \Delta(t)} \tilde{M}_{j} \max \left\{\left\|D_{j}\right\|, \sup _{t_{j}-\tau \leq t<t_{j}} e^{\int_{t_{j}^{\prime}}^{\prime}(\beta(s)-\alpha(s)) d s}\right\}}{e^{\int_{t 0}^{\prime}(\alpha(s)-\beta(s)) d s}}<\infty ;
$$

(2) system (4.1) is asymptotically stable if

$$
\underset{t \rightarrow \infty}{\limsup } \frac{\prod_{j \in \Delta(t)} \tilde{M}_{j} \max \left\{\left\|D_{j}\right\|, \sup _{t_{j}-\tau \leq r<t_{j}} e^{\left.\int_{t_{j}^{\prime}(\beta(s)-\alpha(s)) d s}\right\}}\right.}{e^{\int_{t_{0}}^{\prime}(\alpha(s)-\beta(s)) d s}}=0 ;
$$

(3) if there exists a positive number $\mu$ such that

$$
\limsup _{t \rightarrow \infty} \frac{\prod_{j \in \Delta(t)} \tilde{M}_{j} \max \left\{\left\|D_{j}\right\|, \sup _{t_{j}-t \leq t<t_{j}} e^{\int_{i_{j}(\beta(s)-\alpha(s)) d s}^{\prime}}\right\}}{e^{\int_{t_{0}}^{\prime}(\alpha(s)-\beta(s)+\mu) d s}}=0
$$

then system (4.1) is exponentially stable.

PROOF. By the first part of the proof of Theorem 4.7, we have (4.7). Let

$$
y_{i}(t)=\max _{t_{0}-\tau \leq \xi \leq t}\left\|x_{i}(\xi)\right\| e^{\int_{t_{0}}^{\xi} \alpha(s) d s},
$$

then $y_{i}(t)$ is a nondecreasing function, $i=1, \ldots, r$. As $y_{i}(t)$ is nondecreasing, $\left\|C_{i j}(t)\right\|$ is nonincreasing, and because $l=\sup _{t \geq t_{0}} e^{\int_{--\tau}^{\prime} \alpha(s) d s}<+\infty$, we have

$$
\begin{aligned}
\sum_{j=1}^{r}\left\|C_{i j}(t)\right\|\left\|x_{j}(t-\tau)\right\| e^{\int_{t_{0}}^{\prime} \alpha(s) d s} & \leq \sum_{j=1}^{r}\left\|C_{i j}(t)\right\| y_{j}(t-\tau) e^{\int_{t-\tau}^{\prime} \alpha(s) d s} \\
& \leq \sum_{j=1}^{r}\left\|C_{i j}(t)\right\| l y_{j}(t) .
\end{aligned}
$$

Thus from (4.7), we can get the following estimate:

$$
\left\|x_{i}(t)\right\| e^{\int_{t_{0}}^{\prime} \alpha(s) d s} \leq M_{i}\left\|x_{i t_{0}}\right\|+M_{i} \sum_{j=1}^{r}\left\|C_{i j}\left(t_{0}\right)\right\|\left\|x_{j t_{0}}\right\|+\sum_{j=1}^{r}\left\|C_{i j}(t)\right\| l y_{j}(t)
$$




$$
\begin{aligned}
& +\sum_{j=1}^{r} \int_{t_{0}}^{t}\left(1-\delta_{i j}\right) M_{i}\left\|A_{i j}(s)\right\| y_{j}(s) d s \\
& +\sum_{j=1}^{r} \int_{t_{0}}^{t} M_{i} l L_{i j}(s) y_{j}(s) d s
\end{aligned}
$$

since $y(t)$ is increasing and $\left\|C_{i j}(t)\right\|$ is decreasing. It is easy to see that the right-hand side of the last inequality is increasing and hence

$$
\begin{aligned}
y_{i}(t) \leq & M_{i}\left\|x_{i t_{0}}\right\|+M_{i} \sum_{j=1}^{r}\left\|C_{i j}\left(t_{0}\right)\right\|\left\|x_{j t_{0}}\right\|+\sum_{j=1}^{r}\left\|C_{i j}(t)\right\| l y_{j}(t) \\
& +\sum_{j=1}^{r} \int_{t_{0}}^{t}\left[\left(1-\delta_{i j}\right) M_{i}\left\|A_{i j}(s)\right\|+M_{i} l L_{i j}(s)\right] y_{j}(s) d s .
\end{aligned}
$$

Thus

$$
\begin{aligned}
\sum_{i=1}^{r} y_{i}(t) \leq & \sum_{i=1}^{r} M_{i}\left\|x_{i t_{0}}\right\|+\sum_{i=1}^{r} M_{i} \sum_{j=1}^{r}\left\|C_{i j}\left(t_{0}\right)\right\|\left\|x_{j t_{0}}\right\|+\sum_{i=1}^{r} \sum_{j=1}^{r}\left\|C_{i j}(t)\right\| l y_{j}(t) \\
& +\sum_{i=1}^{r} \sum_{j=1}^{r} \int_{t_{0}}^{t}\left[\left(1-\delta_{i j}\right) M_{i}\left\|A_{i j}(s)\right\|+M_{i} l L_{i j}(s)\right] y_{j}(s) d s \\
\leq & M \sum_{i=1}^{r}\left\|x_{i t_{0}}\right\|+M \sum_{j=1}^{r}\left[\sum_{i=1}^{r}\left\|C_{i j}\left(t_{0}\right)\right\|\right]\left\|x_{j t_{0}}\right\| \\
& +\sum_{j=1}^{r}\left[\sum_{i=1}^{r}\left\|C_{i j}(t)\right\|\right] l y_{j}(t) \\
& +\int_{t_{0}}^{t} M \sum_{j=1}^{r}\left[\sum_{i=1}^{r}\left(1-\delta_{i j}\right)\left\|A_{i j}(s)\right\|+\sum_{i=1}^{r} l L_{i j}(s)\right] y_{j}(s) d s \\
\leq & M\left\|x_{t_{0}}\right\|+M\left\|\tilde{C}\left(t_{0}\right)\right\|\left\|x_{t_{0}}\right\|+\int_{t_{0}}^{t} M[\|\tilde{A}(s)\|+l\|L(s)\|]\|y(s)\| d s \\
& +l\|\tilde{C}(t)\|\|y(t)\| .
\end{aligned}
$$

Since $\|\tilde{C}(t)\|$ is nondecreasing,

$$
\begin{aligned}
\|y(t)\| \leq & M\left\|x_{t_{0}}\right\|\left(1+\left\|\tilde{C}\left(t_{0}\right)\right\|\right)+\int_{t_{0}}^{t} M[\|\tilde{A}(s)\|+l\|L(s)\|]\|y(s)\| d s \\
& +l\left\|\tilde{C}\left(t_{0}\right)\right\|\|y(t)\|
\end{aligned}
$$


and thus

$$
\begin{aligned}
\|y(t)\| & \leq \frac{M\left(1+\left\|\tilde{C}\left(t_{0}\right)\right\|\right)}{1-\left\|\tilde{C}\left(t_{0}\right)\right\|}\left\|x_{t_{0}}\right\|+\frac{M}{1-\left\|\tilde{C}\left(t_{0}\right)\right\|} \int_{t_{0}}^{t}[\|\tilde{A}(s)\|+l\|L(s)\|]\|y(s)\| d s \\
& =\tilde{M}_{0}\left\|x_{t_{0}}\right\|+\int_{t_{0}}^{t} \beta(s)\|y(s)\| d s .
\end{aligned}
$$

The Gronwall-Bellman inequality implies $\|y(t)\| \leq \tilde{M}_{0}\left\|x_{t_{0}}\right\| e^{\int_{t_{0}}^{\prime} \beta(s) d s}$. In view of the relations

$$
\|y(t)\|=\sum_{i=1}^{r} \sup _{s \leq t}\left\|x_{i}(s)\right\| e^{\int_{t_{0}}^{s} \alpha(\eta) d \eta} \geq \sum_{i=1}^{r}\left\|x_{i}(t)\right\| e^{\int_{t_{0}}^{t} \alpha(s) d s}
$$

we obtain

$$
\|x(t)\| \leq \tilde{M}_{0}\left\|x_{t_{0}}\right\| e^{\int_{t_{0}}^{t}[\beta(s)-\alpha(s)] d s}, \quad t \in\left[t_{0}, t_{1}\right)
$$

Using the same argument, it is easy to get that

$$
\|x(t)\| \leq \tilde{M}_{k}\left\|x_{t_{k}}\right\| e^{\int_{k}^{t}[\beta(s)-\alpha(s)] d s}, \quad t \in\left[t_{k}, t_{k+1}\right) .
$$

Furthermore,

$$
\left\|x_{t_{k}}\right\| \leq \tilde{M}_{k-1} \max \left\{\left\|D_{k}\right\|, \sup _{t_{k}-\tau \leq l<t_{k}} e^{\int_{t_{k}}^{\prime}(\beta(s)-\alpha(s)) d s}\right\}\left\|x_{t_{k-1}}\right\| e^{\int_{t_{k-1}}^{t_{k}}(\beta(s)-\alpha(s)) d s} .
$$

Thus when $t \in\left[t_{k}, t_{k+1}\right)$,

$$
\begin{aligned}
\|x(t)\| & \leq \tilde{M}_{k}\left\|x_{t_{k}}\right\| e^{\int_{t_{k}}^{t}[\beta(s)-\alpha(s)] d s} \\
& \leq \tilde{M}_{k} \tilde{M}_{k-1} \max \left\{\left\|D_{k}\right\|, \sup _{t_{k}-\tau \leq t<t_{k}} e^{\int_{t_{k}}^{\prime}(\beta(s)-\alpha(s)) d s}\right\}\left\|x_{t_{k-1}}\right\| e^{\int_{t_{k-1}}^{\prime}(\beta(s)-\alpha(s)) d s} \\
& \leq \cdots \leq \tilde{M}_{0} \prod_{j \in \Delta(t)} \tilde{M}_{j} \max \left\{\left\|D_{j}\right\|, \sup _{t_{j}-\tau \leq t<t_{j}} e^{\int_{I_{j}}^{\prime}(\beta(s)-\alpha(s)) d s}\right\}\left\|x_{t_{0}}\right\| e^{\int_{t_{0}}^{\prime}(\beta(s)-\alpha(s)) d s} .
\end{aligned}
$$

The inequality implies all of the results of Theorem 4.8. The proof is complete.

THEOREM 4.9. Assume that

(1) there exist $l>0, M>1$ and $\alpha \in C[R, R]$ such that $e^{\int_{t-\gamma}^{\prime} \alpha(s) d s} \leq l$ and, for $i=1, \ldots, r,\left\|R_{i i}(t, s)\right\| \leq M e^{\int_{s}^{\prime} \alpha(\eta) d \eta}$;

(2) there exist $c_{i j} \geq 0$ such that $\left\|C_{i j}(t)\right\| \leq c_{i j}, i, j=1, \ldots, r$;

(3) we denote by $\tilde{C}=\left(c_{i j}\right), \tilde{A}(t)=\left(\left\|A_{i j}(t)\right\|\right), L(t)=\left(L_{i j}(t)\right), L_{i j}(t)=$ $\left\|B_{i j}(t)\right\|+\left\|C_{i j}^{\prime}(t)\right\|+\left\|A_{i i}(t)\right\|\left\|C_{i j}(t)\right\|, \tilde{M}=M(1+\|\tilde{C}\|) /(1-\|\tilde{C}\|)$ and $\beta(t)=$ $M(\|\tilde{A}(t)\|+l\|L(t)\|) /(1-\|\tilde{C}\|)$. 
Then

(1) system (4.1) is stable if

$$
\limsup _{t \rightarrow \infty} \frac{\prod_{j \in \Delta(t)} \tilde{M}^{k} \max \left\{\left\|D_{j}\right\|, \sup _{t_{j}-\tau \leq t<t_{j}} e^{\int_{t_{j}}^{\prime}(\beta(s)-\alpha(s)) d s}\right\}}{e^{\int_{t_{0}}^{t}(\alpha(s)-\beta(s)) d s}}<\infty
$$

(2) system (4.1) is asymptotically stable if

$$
\limsup _{t \rightarrow \infty} \frac{\prod_{j \in \Delta(t)} \tilde{M}^{k} \max \left\{\left\|D_{j}\right\|, \sup _{t_{j}-\tau \leq t<t_{j}} e^{\int_{t_{j}}^{t}(\beta(s)-\alpha(s)) d s}\right\}}{e^{\int_{t_{0}}^{\prime}(\alpha(s)-\beta(s)) d s}}=0
$$

(3) if there exists a positive number $\mu$ such that

$$
\limsup _{t \rightarrow \infty} \frac{\prod_{j \in \Delta(t)} \tilde{M}^{k} \max \left\{\left\|D_{j}\right\|, \sup _{t_{j}-\tau \leq t<t_{j}} e^{\int_{t_{j}}^{t}(\beta(s)-\alpha(s)) d s}\right\}}{e^{\int_{t_{0}}^{t}(\alpha(s)-\beta(s)+\mu) d s}}=0,
$$

then system (4.1) is exponentially stable.

PROOF. The proof is similar to that of Theorem 4.8 and thus we omit it here.

THEOREM 4.10. Assume that

(S1) there exist scalar functions $\alpha_{i} \in C\left[I, R^{+}\right]$and constants $c_{i}, M_{i} \geq 1$, such that

$$
\left\|R_{i i}(t, s)\right\| \leq M_{i} e^{-\int_{s}^{\prime} \alpha_{i}(\xi) d \xi} \quad \text { and } \quad \sum_{j=1}^{r}\left\|C_{i j}(t)\right\| \leq c_{i} e^{-\int_{i-\tau}^{t} \alpha_{i}(\xi) d \xi}
$$

$$
\begin{aligned}
& \bar{a}_{i j}(t)=-\delta_{i j} \alpha_{i}(t)+\left(1-\delta_{i j}\right) M_{i}\left\|A_{i j}(t)\right\|, \\
& \bar{b}_{i j}(t)=M_{i}\left\|B_{i j}(t)\right\|+M_{i}\left\|A_{i i}(t)\right\|\left\|C_{i j}(t)\right\|+M_{i}\left\|C_{i j}^{\prime}(t)\right\|,
\end{aligned}
$$

then the stability properties of the system

$$
\begin{cases}\xi_{i}^{\prime}(t)=\sum_{j=1}^{r} \bar{a}_{i j}(t) \xi_{j}(t)+\sum_{j=1}^{r} \bar{b}_{i j}(t) \xi_{j}(t-\tau), & t \in J \backslash I, j=1, \ldots, r, \\ \xi_{i}\left(t_{k}\right)=\sum_{j=1}^{r}\left\|D_{i j}\left(t_{k}\right)\right\| \xi_{j}\left(t_{k}-0\right), & t_{k} \in I,\end{cases}
$$

imply the corresponding stability properties of (4.1).

PROOF. Using the same argument of Theorem 4.7, for $t \in J_{k+1}$, we have

$$
\left\|x_{i}(t)\right\| \leq M_{i} e^{-\int_{i_{k}} \alpha_{i}(s) d s}\left\|x_{t_{k}}\right\|+\sum_{j=1}^{r} \int_{t_{k}}^{t}\left(1-\delta_{i j}\right) M_{i} e^{-\int_{\xi} \alpha_{i}(s) d s}\left\|A_{i j}(\xi)\right\|\left\|x_{j}(\xi)\right\| d \xi
$$




$$
\begin{aligned}
& +\sum_{j=1}^{r} \int_{t_{k}}^{t} M_{i} e^{-\int_{k}^{\prime} \alpha_{i}(s) d s}\left\|B_{i j}(\xi)\right\|\left\|x_{j}(\xi-\tau)\right\| d \xi \\
& +M_{i} e^{-\int_{i_{k}}^{t} \alpha_{i}(s) d s} c_{i}\left\|x_{t_{k}}\right\|+c_{i} e^{-\int_{i_{k}}^{t} \alpha_{i}(s) d s}\left\|x_{t_{k}}\right\| \\
& +\sum_{j=1}^{r} \int_{t_{k}}^{t} M_{i} e^{-\int_{k}^{t} \alpha_{i}(s) d s}\left\|A_{i i}(\xi)\right\|\left\|C_{i j}(\xi)\right\|\left\|x_{j}(\xi-\tau)\right\| d \xi \\
& +\sum_{j=1}^{r} \int_{t_{k}}^{t} M_{i} e^{-\int_{k}^{\prime} \alpha_{i}(s) d s}\left\|C_{i j}^{\prime}(\xi)\right\|\left\|x_{j}(\xi-\tau)\right\| d \xi \\
& =: P_{i}(t) .
\end{aligned}
$$

Then $\left\|x_{i}(t)\right\| \leq P_{i}(t), t \in J_{k+1}$. Let $P_{i}\left(t_{k}\right)=\sum_{j=1}^{r}\left\|D_{i j}\left(t_{k}\right)\right\| P_{j}\left(t_{k}-0\right), t_{k} \in I$, then $\left\|x_{i}(t)\right\| \leq P_{i}(t), t \geq t_{0}$. Furthermore,

$$
\begin{aligned}
P_{i}^{\prime}(t) \leq & -\alpha_{i}(t) P_{i}(t)+\sum_{j=1}^{r}\left(1-\delta_{i j}\right) M_{i}\left\|A_{i j}(t)\right\|\left\|x_{j}(t)\right\| \\
& +\sum_{j=1}^{r} M_{i}\left\|B_{i j}(t)\right\|\left\|x_{j}(t-\tau)\right\|+\sum_{j=1}^{r} M_{i}\left\|A_{i i}(t)\right\|\left\|C_{i j}(t)\right\|\left\|x_{j}(t-\tau)\right\| \\
& +\sum_{j=1}^{r} M_{i}\left\|C_{i j}^{\prime}(t)\right\|\left\|x_{j}(t-\tau)\right\| \\
\leq & -\alpha_{i}(t) P_{i}(t)+\sum_{j=1}^{r}\left(1-\delta_{i j}\right) M_{i}\left\|A_{i j}(t)\right\| P_{j}(t)+\sum_{j=1}^{r} M_{i}\left\|B_{i j}(t)\right\| P_{j}(t-\tau) \\
& +\sum_{j=1}^{r} M_{i}\left\|A_{i i}(t)\right\|\left\|C_{i j}(t)\right\| P_{j}(t-\tau)+\sum_{j=1}^{r} M_{i}\left\|C_{i j}^{\prime}(t)\right\| P_{j}(t-\tau) \\
= & \sum_{j=1}^{r} \bar{a}_{i j}(t) P_{j}(t)+\sum_{j=1}^{r} \bar{b}_{i j}(t) P_{j}(t-\tau), \quad t \in J_{k+1} .
\end{aligned}
$$

Let $P(t)=\operatorname{col}\left(P_{1}(t), \ldots, P_{r}(t)\right), P_{i}\left(t_{k}\right)=\sum_{j=1}^{r}\left\|D_{i j}\left(t_{k}\right)\right\| P_{j}\left(t_{k}-0\right)$, then $\left\|x_{i}(t)\right\| \leq$ $P_{i}(t), t \in J$. Consider the comparison system

$$
\begin{aligned}
& \begin{cases}P_{i}^{\prime}(t) \leq \sum_{j=1}^{r} \bar{a}_{i j}(t) P_{j}(t)+\sum_{j=1}^{r} \bar{b}_{i j}(t) P_{j}(t-\tau), & t_{k} \in J \backslash I, \\
P_{i}\left(t_{k}\right)=\sum_{j=1}^{r}\left\|D_{i j}\left(t_{k}\right)\right\| P_{j}\left(t_{k}-0\right), & t_{k} \in I ;\end{cases} \\
& \begin{cases}\xi_{i}^{\prime}(t)=\sum_{j=1}^{r} \bar{a}_{i j}(t) \xi_{j}(t)+\sum_{j=1}^{r} \bar{b}_{i j}(t) \xi_{j}(t-\tau), & t \in J \backslash, \\
\xi_{i}\left(t_{k}\right)=\sum_{j=1}^{r}\left\|D_{i j k}\right\| \xi_{j}\left(t_{k}-0\right), & t_{k} \in I .\end{cases}
\end{aligned}
$$

Lemma 2.2 and $\|x(t)\| \leq\|P(t)\|$ imply that $\|x(t)\| \leq\|\xi(t)\|$, which implies that the conclusions of the theorem are true. The proof is complete. 
EXAMPLE 3. Consider the neutral impulsive system

$$
\left\{\begin{aligned}
\frac{d x_{1}(t)}{d t}= & A_{11}(t) x_{1}(t)+A_{12}(t) x_{2}(t)+B_{11}(t) x_{1}(t-1) \\
& +C_{12}(t) x_{2}^{\prime}(t-1) \\
\frac{d x_{2}(t)}{d t}= & A_{21}(t) x_{1}(t)+A_{22}(t) x_{2}(t)+B_{22}(t) x_{2}(t-1) \\
& +C_{21}(t) x_{1}^{\prime}(t-1), \quad t \neq t_{k}, \\
x\left(t_{k}\right)= & D_{k} x\left(t_{k}-0\right), \quad k=1,2, \ldots,
\end{aligned}\right.
$$

where $t_{0} \geq 0, x=\left(x_{1}, x_{2}\right)^{T} \in R^{4}, x_{1}, x_{2} \in R^{2}, D_{k}=\left(D_{i j k}\right) \in R^{4 \times 4}, D_{i j k} \in R^{2 \times 2}$, $i, j=1,2$ and

$$
\begin{array}{ll}
A_{11}(t)=\left[\begin{array}{cc}
-4 & \left(1+t^{2}\right)^{-1} \\
-\left(1+t^{2}\right)^{-1} & -4
\end{array}\right], & A_{12}(t)=\left[\begin{array}{cc}
\left(\cos ^{2} t\right) / 2 & 1 / 6 \\
\left(\sin ^{2} t\right) / 2 & \left(\cos ^{2} t\right) / 4
\end{array}\right], \\
A_{21}(t)=\left[\begin{array}{cc}
1 / 4 & 0 \\
1 / 14 & -1 / 3
\end{array}\right], & A_{22}(t)=\left[\begin{array}{cc}
-4-\cos ^{2} t & \sin ^{2} t \\
-\sin ^{2} t & -4
\end{array}\right], \\
B_{11}(t)=\left[\begin{array}{cc}
(4 e)^{-1} & {\left[4 e\left(1+t^{2}\right)\right]^{-1}} \\
0 & 0
\end{array}\right], & B_{22}(t)=\left[\begin{array}{cc}
0 & \left(4 e^{2}\right)^{-1} \\
(4 e)^{-1} & 0
\end{array}\right], \\
C_{12}(t)=\left[\begin{array}{cc}
e^{-4(t+1)} / 2 & 0 \\
0 & e^{-4(t+1)} / 2
\end{array}\right], & C_{21}(t)=\left[\begin{array}{cc}
\left(4 e^{4(t+1)}\right)^{-1} & 0 \\
\left(9 e^{3(t+1)}\right)^{-1} & 0
\end{array}\right], \\
C_{12}^{\prime}(t)=\left[\begin{array}{cc}
-4 e^{-4(t+1)} & 0 \\
0 & -2 e^{-4(t+1)}
\end{array}\right], & C_{21}^{\prime}(t)=\left[\begin{array}{cc}
-\left(e^{4(t+1)}\right)^{-1} & 0 \\
-\left(3 e^{3(t+1)}\right)^{-1} & 0
\end{array}\right] .
\end{array}
$$

Then the fundamental matrix solutions $R_{i i}\left(t, t_{0}\right)$ and $R_{22}\left(t, t_{0}\right)$ of systems $x_{1}^{\prime}(t)=$ $A_{11}(t) x_{1}(t)$ and $x_{2}^{\prime}(t)=A_{22}(t) x_{2}(t)$ satisfy

$$
\left\|R_{11}\left(t, t_{0}\right)\right\| \leq \sqrt{2} e^{-4\left(t-t_{0}\right)}, \quad\left\|R_{22}\left(t, t_{0}\right)\right\| \leq \sqrt{2} e^{-3\left(t-t_{0}\right)}
$$

and

$$
\begin{aligned}
& \left\|A_{11}(t)\right\| \leq 5, \quad\left\|A_{22}(t)\right\| \leq 5, \quad\left\|A_{12}(t)\right\|=1 / 2, \quad\left\|A_{21}(t)\right\| \leq 1 / 3, \\
& \left\|B_{11}(t)\right\| \leq(4 e)^{-1}, \quad\left\|B_{22}(t)\right\| \leq(4 e)^{-1}, \quad\left\|C_{12}(t)\right\| \leq e^{-4(t+1)} / 2, \\
& \left\|C_{21}(t)\right\| \leq e^{-4(t+1)} \quad \text { and } \quad\left\|B_{12}\right\|=\left\|B_{21}\right\|=\left\|C_{11}\right\|=\left\|C_{22}\right\|=0 .
\end{aligned}
$$

Using the notation of Theorem 4.10 and a simple argument, we have

$$
\begin{gathered}
t_{k}=k, \quad \tau=1, \quad \alpha_{1}=4, \quad \alpha_{2}=3, \quad M_{1}=M_{2}=\sqrt{2}, \\
\tilde{a}_{11}(t)=-\alpha_{1}=-4, \quad \tilde{a}_{12}(t)=M_{1}\left\|A_{12}(t)\right\| \leq \sqrt{2} / 2, \\
\tilde{a}_{21}(t)=M_{2}\left\|A_{21}(t)\right\| \leq \sqrt{2} / 3, \quad \tilde{a}_{22}(t)=-\alpha_{2}=-3, \\
\tilde{b}_{11}(t) \leq(2 e)^{-1}, \quad \tilde{b}_{12}(t) \leq 1 / e, \quad \tilde{b}_{21}(t) \leq(2 e)^{-1} \quad \text { and } \quad \tilde{b}_{22}(t) \leq 1 / e .
\end{gathered}
$$


Let the comparison system be

$$
\left\{\begin{array}{l}
\xi^{\prime}(t)=\left[\begin{array}{cc}
-4 & \sqrt{2} / 2 \\
\sqrt{2} / 3 & -3
\end{array}\right] \xi(t)+\left[\begin{array}{cc}
1 / 2 e & 1 / 2 e \\
1 / 2 e & 1 / 2 e
\end{array}\right] \xi(t-1), \quad t \neq t_{k}, \\
\xi\left(t_{k}\right)=\left(\left\|D_{i j k}\right\|\right)_{2 \times 2} \xi\left(t_{k}-0\right) .
\end{array}\right.
$$

From (4.10), using the notation of Theorem 3.1, we have $\alpha_{1}=4, \alpha_{2}=3, c_{1}=c_{2}=1$, $\gamma=2.1,\|\tilde{B}\|=e^{-1}, \tau=1$ and $\alpha=1$. Let

$$
D_{k}=\left[\begin{array}{cc}
D_{11 k} & D_{12 k} \\
D_{21 k} & D_{22 k}
\end{array}\right]=\left[\begin{array}{cccc}
e-3 / 2 & 0 & 0 & 0 \\
1 / 2 & 0 & 0 & 0 \\
0 & 1 & 1 / 2 & 0 \\
0 & 0 & 0 & 1
\end{array}\right]
$$

then $\left\|D_{k}\right\|=\left\|\tilde{D}_{k}\right\|=e-1$ and

(1) if $t_{k}-t_{k-1} \geq \tau=1$, then $e^{-t} \prod_{j \in \Delta(t)} \max \left\{\left\|\tilde{D}_{j}\right\|, e^{\alpha \tau}\right\} \leq 1$,

(2) if $t_{k}-t_{k-1} \geq \tau+1 / k$, then $\lim _{t \rightarrow \infty}\left(e^{-t} \prod_{j \in \Delta(t)} \max \left\{\left\|\tilde{D}_{j}\right\|, e^{\alpha \tau}\right\}\right)=0$,

(3) if $t_{k}-t_{k-1} \geq \tau+\eta, \eta>0$, then $e^{-t /(1+\eta)} \prod_{j \in \Delta(t)} \max \left\{\left\|\tilde{D}_{j}\right\|, e^{\alpha \tau}\right\} \leq e$.

By Theorem 4.4, system (4.9) is stable in case (1); asymptotically stable in case (2); and exponentially stable in case (3).

\section{Conclusion}

In this paper, we have studied the stability issue for both linear and nonlinear impulsive functional systems with delay. Our approach has utilised the comparison principle and an inequality for the establishment of stability criteria. Although only a single delay has been considered in this paper, the study can be extended to the case with multiple delays.

\section{Acknowledgements}

The authors wish to thank the referees whose comments improved the quality of the paper. This work was supported by the Natural Sciences and Engineering Research Council of Canada.

\section{References}

[1] A. Anokhin, L. Berezansky and E. Braverman, "Exponential stability of linear delay impulsive differential equations". J. Math. Anal. Appl. 193 (1995) 923-941. 
[2] G. Ballinger and X. Liu, "On boundedness of solutions of impulsive systems", Nonlinear Stud. 4 (1997) 121-131.

[3] G. Ballinger and X. Liu, "Existence and uniqueness results for impulsive delay differential equations", Dynam. Contin. Discrete Impuls. Systems 5 (1999) 579-591.

[4] L. Berezansky and L. Idels, "Exponential stability of some scalar impulsive delay differential equation", Commun. Appl. Anal. 2 (1998) 301-309.

[5] S. G. Deo and S. G. Pandit, Differential systems involving impulses, Lecture Notes 954 (Springer, Berlin, 1982).

[6] Z. Guan, "Decentralized stabilization for impulsive large-scale systems with delays", Dynam. Contin. Discrete Impuls. Systems 6 (1999) 367-379.

[7] D. Guo and X. Liu, "First order impulsive integro-differential equations on unbounded domain in a Banach space", Dynam. Contin. Discrete Impuls. Systems 2 (1996) 381-394.

[8] S. V. Krishna and A. V. Anokhin, "Delay differential systems with discontinuous initial data and existence and uniqueness theorems for systems with impulse and delay", J. Appl. Math. Stochastic Anal. 7 (1994) 49-67.

[9] V. Lakshmikantham, D. D. Bainov and P. S. Simeonov, Theory of impulsive differential equations (World Scientific, Teaneck, NJ, 1989).

[10] V. Lakshmikantham and S. Leela, Differential and integral inequalities (Academic Press, New York, 1969).

[11] V. Lakshmikantham and X. Liu, "Stability criteria for impulsive differential equations in term of two measures", J. Math. Anal. Appl. 137 (1989) 591-604.

[12] X. Liu, "Stability results for impulsive differential systems with applications to population growth models", Dynam. Stability Systems 9 (1994) 163-174.

[13] X. Liu and X. Liao, "Comparison method and robust stability of large-scale dynamic systems", Dynam. Contin. Discrete Impuls. Systems Ser. A Math. Anal. 11 (2004) 413-430.

[14] X. Liu and J. Shen, "Asymptotic behavior of solutions of impulsive neutral differential equations", Appl. Math. Lett. 12 (1999) 51-58.

[15] V. D. Mil'man and A. D. Myshkis, "On the stability of motion in the presence of impulses", Siberian Math. J. 1 (1960) 233-237.

[16] T. Pavlids, "Stability of systems described by differential equations containing impulses", IEEE Trans. Automatic Control 12 (1967) 43-45.

[17] J. Shen, Z. Luo and X. Liu, "Impulsive stabilization of functional differential equations via Liapunov functionals", J. Math. Anal. Appl. 240 (1999) 1-15.

[18] J. Shen and J. Yan, "Razumikhim type stability theorems for impulsive functional differential equations", Nonlinear Anal. 33 (1998) 519-531.

[19] L. Wen and P. Weng, "Weakly exponentially asymptotic stability of functional differential equation with impulses", Dynam. Contin. Discrete Impuls. Systems 6 (1999) 251-269.

[20] T. Yang, Impulsive control theory, Lecture Notes in Control and Information Sciences 272 (Springer, Berlin, 2000). 